\title{
Influence of Strouhal number on pulsating methane-air coflow jet diffusion flames
}

\author{
M. Sánchez-Sanz, ${ }^{a *}$ B.A.V. Bennett, ${ }^{b}$ M.D. Smooke ${ }^{b}$ and A. Liñán ${ }^{\mathrm{a}}$ \\ ${ }^{a}$ Departamento de Motopropulsión y Termofluidomecánica, ETSI Aeronáuticos, Universidad \\ Politécnica de Madrid, Madrid, Spain; ${ }^{b}$ Department of Mechanical Engineering, Yale University, \\ New Haven, CT, USA
}

(Received 16 July 2009; final version received 30 March 2010)

\begin{abstract}
Four periodically time-varying methane-air laminar coflow jet diffusion flames, each forced by pulsating the fuel jet's exit velocity $U_{j}$ sinusoidally with a different modulation frequency $w_{j}$ and with a $50 \%$ amplitude variation, have been computed. Combustion of methane has been modeled by using a chemical mechanism with 15 species and 42 reactions, and the solution of the unsteady Navier-Stokes equations has been obtained numerically by using a modified vorticity-velocity formulation in the limit of low Mach number. The effect of $w_{j}$ on temperature and chemistry has been studied in detail. Three different regimes are found depending on the flame's Strouhal number $S=a w_{j} / U_{j}$, with $a$ denoting the fuel jet radius. For small Strouhal number $(S=0.1)$, the modulation introduces a perturbation that travels very far downstream, and certain variables oscillate at the frequency imposed by the fuel jet modulation. As the Strouhal number grows, the nondimensional frequency approaches the natural frequency of oscillation of the flickering flame ( $S \simeq 0.2$ ). A coupling with the pulsation frequency enhances the effect of the imposed modulation and a vigorous pinch-off is observed for $S=0.25$ and $S=0.5$. Larger values of $S$ confine the oscillation to the jet's near-exit region, and the effects of the pulsation are reduced to small wiggles in the temperature and concentration values. Temperature and species mass fractions change appreciably near the jet centerline, where variations of over $2 \%$ for the temperature and $15 \%$ and $40 \%$ for the $\mathrm{CO}$ and $\mathrm{OH}$ mass fractions, respectively, are found. Transverse to the jet movement, however, the variations almost disappear at radial distances on the order of the fuel jet radius, indicating a fast damping of the oscillation in the spanwise direction.
\end{abstract}

Keywords: pulsating flames; full chemistry; maximum flame temperature; CO oxidation; flame pinch-off

\section{Introduction}

In most practical applications, combustion is a complicated unsteady turbulent process that is difficult to model. The ability to predict the interaction between the fluid dynamics and the chemical reaction is fundamental to an understanding of important phenomena such as extinction, vortex-flame interaction, and pollutant formation in diffusion flames. Unfortunately, because technical difficulties increase as the complexity of the chemistry 
increases, the Reynolds number of such flows is often reduced and unsteadiness is kept to a minimum.

Unsteady combustion has been of interest since the beginning of the last century, as reported in the 1928 study of Chamberlin and Rose [1], where an estimation of the frequency of oscillation of unforced diffusion flames was provided. This study was later continued during the 1970s and 1980s by Ballantyne and Bray [2], Beker and Liang [3], and Grant and Jones [4], among others, who reported that the instabilities that cause the flame to flicker under normal gravity conditions can lead to the quasi-periodic break-up of the flame. During the same two decades, several authors considered the idea of exciting a flow at a specific frequency to study the formation of large eddies in cold flows; see the excellent review of Perry and Chong [5]. In Strawa and Cantwell [6,7], this idea was used for the first time to excite a low-speed coaxial jet diffusion flame at low Reynolds number. They found that, by imposing a perturbation at a certain frequency, it was possible to produce a completely periodic, fully controllable flow that allowed the modification of the chemical reaction and the mixing process. The same conclusion was also later suggested by Lovett and Turns $[8,9]$ for turbulent jets.

The ideas described above, which were derived experimentally for forced nonreacting jets, have been revisited recently in the context of computations and theory. For heated jets, Jiang et al. [10] in 2004 discovered faster downstream decay rates for the unsteady component of the velocity than in the isothermal problem. According to the 2006 numerical work of Barve et al. [11], the oscillation frequency is the critical parameter for the downstream evolution of the jet, indicating that for small frequencies of oscillation the effects of the pulsation are convected far downstream, while for large frequencies the effects of the pulsation are confined to the jet's near-exit region. Earlier this year, Riley et al. [12] derived an expression for the velocity decay rate of a Bickley jet perturbed by an oscillatory component of small amplitude. They found

$$
u \sim S^{-1} x^{-2 / 3} \exp \left[-C_{1} S^{1 / 2}(\nu z)^{2 / 3}\right] \cos \left[t-C_{2} S(\nu z)^{2 / 3}\right]
$$

for the centerline unsteady velocity component, with the Strouhal number defined by $S=a w_{j} / U_{j}$, where $a$ is the initial jet radius, $w_{j}$ the oscillation frequency, and $U_{j}$ the characteristic velocity. In Equation (1), $v$ is the nondimensional viscosity, $z$ the downstream distance, $t$ the time, and $C_{1}$ and $C_{2}$ constants whose values are known. For constant Reynolds number $R e$, the time-dependent perturbation shows a fast exponential decay with downstream axial distance, in contrast to the slow algebraic decay of the timeaveraged solution, which ends up developing into the well-known Bickley jet solution [13].

Also relevant to the present work are previous investigations that have examined unsteady coflowing methane-air flames, but not necessarily with a focus on spatial or temporal decay rates [14-21]. All of these investigations date from the last two decades. Both the 1993 experimental study of Smyth et al. [14] and the 1996 computational/experimental comparisons of Kaplan et al. [15] noted enhanced soot production in flames that were sinusoidally forced at $10 \mathrm{~Hz}$; the computations involved a five-species chemical model and a two-equation soot model. Also in 1996, Skaggs and Miller [16] measured CO and temperature profiles in a forced flame generated on a burner having the same dimensions and flowrates as that in $[14,15]$. They determined that the time-averaged $\mathrm{CO}$ concentrations were higher than for the corresponding steady flame. In 1998, Mohammed et al. [17] performed a computational and experimental study of a flame forced at $20 \mathrm{~Hz}$ and found that species indicative of soot production had higher peak values in the time-varying flame than 
in the corresponding steady flame. The governing equations in [17] were written using a primitive variable formulation, the chemistry was modeled using a 26-species mechanism, and the solution was obtained via an implicit Newton-based algorithm. Pember et al. [18] and Day and Bell [19] computed an unforced flickering flame (two-step reaction mechanism) and a $20-\mathrm{Hz}$ forced flame (26-species reaction mechanism), respectively; both studies used semi-implicit projection methods combined with adaptive mesh refinement to solve the primitive variable formulation of the governing equations. Finally, the 2001 study of Bennett and Smooke [20] (which employed a 31-species C2 mechanism) and the 2007 work of Dworkin et al. [21] (which employed a 16-species C1 mechanism) both examined flames forced at $20 \mathrm{~Hz}$ using the vorticity-velocity formulation and an implicit Newton-based algorithm; both studies compared temperature and species profiles with experimental data. The purpose of all of these previous studies of unsteady coflowing methane-air flames was either to show that a numerical method produced "reasonable" results, to validate numerical models and methods by demonstrating good comparisons with experimental measurements, or to examine the enhancement of a particular quantity (e.g., soot) during unsteady combustion. While some of these studies [19-21] involved more than one amplitude of forcing perturbation, none of them examined flame behavior for multiple forcing frequencies.

In this paper we investigate the structure of a detailed chemistry, axisymmetric, coflow diffusion flame in which the fuel jet is pulsated with a given amplitude and an imposed frequency; flame behavior is examined at various frequencies. The Reynolds number, based upon the fuel jet radius, is moderately small $(R e \sim 100)$, so the flow remains laminar. In the next section, we formulate the problem; in Section 3, we introduce the numerical method used in computing the solutions; and in Section 4, we present the results. Specifically, we confirm the experimental observations of Strawa and Cantwell [6,7] and Lovett and Turns $[8,9]$ and extend their explanation using some recent asymptotic and numerical work to analyze the influence of the pulsation on the maximum flame temperature, flame shape, and $\mathrm{CO}$ oxidation chemistry. In Section 5, we summarize the main conclusions of the work.

\section{Problem formulation}

The problem of a diluted methane fuel jet composed of $65 \% \mathrm{CH}_{4}$ and $35 \% \mathrm{~N}_{2}$, by mole, discharging into an air coflow with a jet velocity that changes periodically with time (Figure 1) is modeled using the modified vorticity-velocity formulation described in [22]. In the commonly employed version of the vorticity-velocity equations, mass loss and gain have been reported in flows with large vorticity gradients. The reason for this spurious behavior resides in the derivation of the equations, in which the derivative of the continuity equation is satisfied, but not the equation itself. This problem has been solved by substituting the kinematic definition of vorticity in certain terms of the fluid-dynamic equations [22] without changing the elliptic character of the equations - a property that is known to favor convergence of a fully implicit solver such as the Newton-based solver described in Section 3

The full set of equations is made nondimensional by using characteristic values of the variables at the jet exit: density $\rho_{j}$; dynamic viscosity $\mu_{j}$; specific heat at constant pressure $c_{p_{j}}$; temperature $T_{j}\left(298 \mathrm{~K}\right.$ ); and velocity $U_{j}$ (peak velocity in the parabolic profile, before fluctuation is imposed). As a characteristic length, we use the jet radius $a$. Introducing these scales, the nondimensionalized equations are written as follows. 


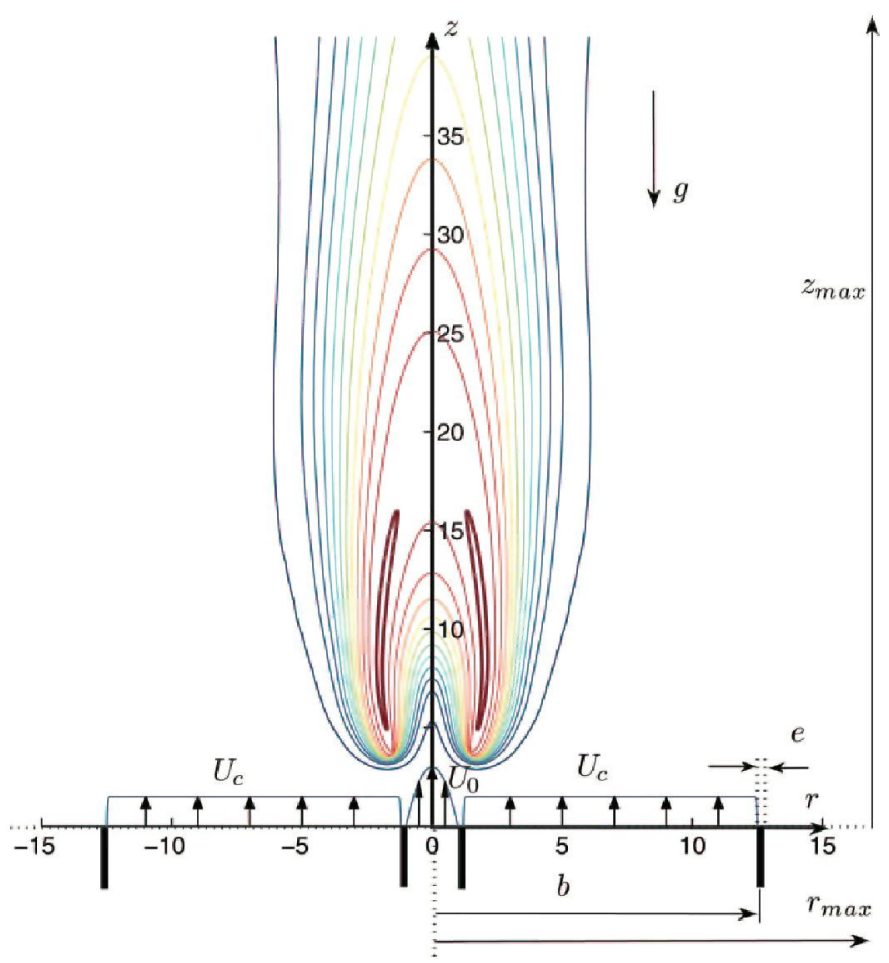

Figure 1. Coordinate system and geometry considered in the calculations. The acceleration due to gravity acts downward, toward decreasing values of the $z$ coordinate. This sample calculation shows equally-spaced temperature isotherms $(\Delta T=0.5)$ for the steady problem obtained by imposing $S=0$ in Equations (2)-(12). The thick lines indicate the maximum plotted temperature $T=6.5$. The shapes and relative magnitudes of the inlet velocity profiles are indicated at the bottom of the figure.

- Radial velocity equation

$$
S \frac{\partial}{\partial r}\left(\frac{1}{\rho} \frac{\partial \rho}{\partial t}\right)+\frac{\partial^{2} v_{r}}{\partial r^{2}}+\frac{\partial^{2} v_{r}}{\partial z^{2}}=\frac{\partial w}{\partial z}-\frac{1}{r} \frac{\partial v_{r}}{\partial r}+\frac{v_{r}}{r^{2}}-\frac{\partial}{\partial r}\left(\frac{\vec{v} \cdot \nabla \rho}{\rho}\right)
$$

- Axial velocity equation

$$
S \frac{\partial}{\partial z}\left(\frac{1}{\rho} \frac{\partial \rho}{\partial t}\right)+\frac{\partial^{2} v_{z}}{\partial z^{2}}=\frac{\partial^{2} v_{r}}{\partial r \partial z}-\frac{1}{r} \frac{\partial v_{r}}{\partial z}-\frac{\partial}{\partial z}\left(\frac{\vec{v} \cdot \nabla \rho}{\rho}\right)
$$

Here, $\rho$ is the density of the mixture, $v_{r}$ is the radial velocity, $v_{z}$ is the axial velocity, $\omega=a \omega^{\prime} / U_{j}=\partial v_{r} / \partial z-\partial v_{z} / \partial r$ is the vorticity, $t=w_{j} t^{\prime}$ is the time, and $z=z^{\prime} / a$ and $r=r^{\prime} / a$ are the axial and transverse (radial) coordinates, respectively. The Strouhal number is defined as $S=a w_{j} / U_{j}$, with $w_{j}$ the frequency of oscillation of the jet velocity. Here and hereafter, the prime ( $\left.{ }^{(}\right)$denotes dimensional quantities. 


$$
\begin{aligned}
S\left(\rho \frac{\partial \omega}{\partial t}-\omega \frac{\partial \rho}{\partial t}+\bar{\nabla} \rho \cdot \frac{\partial \vec{v}}{\partial t}\right)+\rho v_{r} \frac{\partial \omega}{\partial r}+\rho v_{z} \frac{\partial \omega}{\partial z} \\
=\frac{\rho v_{r}}{r}\left(\frac{\partial v_{r}}{\partial z}-\frac{\partial v_{z}}{\partial r}\right)-\bar{\nabla} \rho \cdot \nabla\left(\frac{\vec{v} \cdot \vec{v}}{2}\right)+\frac{1}{F r^{2}} \frac{\partial \rho}{\partial r} \\
\quad-\frac{1}{R e}\left\{2\left[\bar{\nabla}(\nabla \cdot \vec{v}) \cdot \nabla \mu-\nabla v_{r} \cdot \bar{\nabla}\left(\frac{\partial \mu}{\partial r}\right)-\nabla v_{z} \cdot \bar{\nabla}\left(\frac{\partial \mu}{\partial z}\right)\right]\right. \\
\left.\quad-\frac{\partial^{2}(\mu \omega)}{\partial r^{2}}-\frac{\partial^{2}(\mu \omega)}{\partial z^{2}}-\frac{\partial}{\partial r}\left(\frac{\mu}{r} \frac{\partial v_{r}}{\partial z}\right)+\frac{\partial}{\partial r}\left(\frac{\mu}{r} \frac{\partial v_{z}}{\partial r}\right)\right\}
\end{aligned}
$$

Here, the operator $\bar{\nabla} \beta=(\partial \beta / \partial z,-\partial \beta / \partial r)$ has been defined for convenience for any scalar $\beta$. The nondimensional mixture viscosity $\mu$ is calculated through Wilke's expression [23], while the viscosity of every species is obtained by using the curvefit coefficients given in [24]. Based on the given composition of the fuel jet, the Froude and Reynolds numbers are calculated to be $F r=U_{j} / \sqrt{a g}=5$ and $R e=U_{j} a / v_{j}=90$, respectively.

- Energy equation

$$
\begin{gathered}
S \rho c_{p} \frac{\partial T}{\partial t}+\rho c_{p}\left(v_{r} \frac{\partial T}{\partial r}+v_{z} \frac{\partial T}{\partial z}\right)=\frac{1}{\operatorname{Re} \operatorname{Pr}}\left[\frac{1}{r} \frac{\partial}{\partial r}\left(r \mu c_{p} \frac{\partial T}{\partial r}\right)+\frac{\partial}{\partial z}\left(\mu c_{p} \frac{\partial T}{\partial z}\right)\right] \\
+\frac{1}{\operatorname{Re}} \sum_{\substack{n=1 \\
n \neq \mathrm{N}_{2}}}^{N_{s}} \frac{c_{p_{n}}-c_{p_{\mathrm{N}_{2}}}}{S c_{n}} \mu \nabla Y_{n} \cdot \nabla T-\sum_{n=1}^{N_{s}} h_{n} \dot{w}_{n}-\nabla \cdot \vec{q}_{\mathrm{R}}+\frac{E c}{\operatorname{Re}} \Omega_{v}
\end{gathered}
$$

In the above equation, $N_{s}$ represents the number of species in the mechanism. The nondimensional radiative flux $\nabla \cdot \overrightarrow{q_{\mathrm{R}}}$ is calculated using an optically thin radiation model with $\mathrm{H}_{2} \mathrm{O}, \mathrm{CO}$, and $\mathrm{CO}_{2}$ as radiating species [25], and $\Omega_{v}$ is the viscous dissipation term, given by

$$
\Omega_{v}=\mu\left[2\left(\frac{\partial v_{r}}{\partial r}\right)^{2}+2\left(\frac{v_{r}}{r}\right)^{2}+2\left(\frac{\partial v_{z}}{\partial z}\right)^{2}+\left(\frac{\partial v_{r}}{\partial z}+\frac{\partial v_{z}}{\partial r}\right)^{2}-\frac{2}{3}(\nabla \cdot \vec{v})^{2}\right]
$$

The Eckert number $E c=U_{j}^{2} /\left(c_{p_{j}} T_{j}\right) \sim 0.082$ is very small and anticipates the small influence of the viscous dissipation term in the calculations. The specific heat at constant pressure of the mixture is calculated by using $c_{p}=\sum_{n=1}^{N_{s}} Y_{n} c_{p_{n}}$, with $c_{p_{n}}$ representing the specific heat of the $n$th species at constant pressure, obtained via the polynomial approximation given in [24]. The nondimensional enthalpy is defined as $h_{n}=h_{n}^{\prime} /\left(c_{p_{j}} T_{j}\right)$, while $\dot{w}_{n}=\dot{w}_{n}^{\prime} a W_{n} /\left(\rho_{j} U_{j}\right)$ is the net rate of production of the $n$th species, with $W_{n}$ the molecular weight of the $n$th species. In writing the energy equation, we have neglected the Dufour effect and have assumed a Fickian diffusion law, so that $Y_{n} \vec{V}_{n}=-D_{n} \nabla Y_{n}$ for $n \neq \mathrm{N}_{2}$, where $\vec{V}_{n}$ is the diffusion velocity of the $n$th species and $D_{n}$ is the diffusion coefficient of the $n$th species. Similar to what has been done previously [26-28], we impose a constant Prandtl number, $\operatorname{Pr}=\mu c_{p} / \lambda$, where $\lambda$ denotes the thermal conductivity. The approximate value for air, $\operatorname{Pr}=0.7$, is employed throughout the study. The reference values for the diffusion coefficients $D_{n}$ were obtained from [28] so that the 
Schmidt number $S c_{n}=\operatorname{Pr} L e_{n}$ is constant. Lewis numbers $L e_{n}=\lambda /\left(c_{p} \rho D_{n}\right)$ for the different species can also be found in [28].

- Species equation (for $n=1, \ldots, N_{s}, n \neq \mathrm{N}_{2}$ )

$$
S \rho \frac{\partial Y_{n}}{\partial t}+\rho v_{r} \frac{\partial Y_{n}}{\partial r}+\rho v_{z} \frac{\partial Y n}{\partial x}=\frac{1}{S c_{n} \operatorname{Re}}\left[\frac{1}{r} \frac{\partial}{\partial r}\left(\rho r \mu \frac{\partial Y_{n}}{\partial r}\right)+\frac{\partial}{\partial z}\left(\mu \frac{\partial Y_{n}}{\partial r}\right)\right]+\dot{w}_{n}
$$

- Excess species equation

$$
Y_{N_{2}}=1-\sum_{\substack{n=1 \\ n \neq \mathrm{N}_{2}}}^{N_{s}} Y_{n}
$$

Since the Mach number of the flow is small, pressure derivatives can be neglected in the energy equation, and the pressure remains constant and equal to atmospheric pressure $(p=1)$. The density of the mixture can therefore be calculated from the ideal gas law as

$$
\rho=\frac{\bar{W}}{T}
$$

where $\bar{W}=\bar{W}^{\prime} / \bar{W}_{j}=\left(\sum_{n=1}^{N_{s}} Y_{n} / W_{n}\right)^{-1}$ is the mean molecular weight of the gas mixture, and $\bar{W}_{j}=20.198 \mathrm{~g} / \mathrm{mol}$ is the mean molecular weight at the fuel jet exit. The chemical reaction terms in Equations (5) and (6) are modeled by using a finite-rate C1-chemistry mechanism composed of 42 reversible reaction steps and $N_{s}=15$. Modified Arrhenius expressions are used for forward and backward rate constants, whose parameters can be found in [29].

The system of equations presented above must be complemented with suitably defined boundary conditions on each side of the computational domain. The domain covers an area extending from $r=0$ to $r=r_{\max }=38$ and $z=0$ to $z=z_{\max }=100$. The thickness of the fuel tube wall is set at $e=0.19$ and is chosen to mimic the experimental set-up in [21]. The boundary conditions are given below.

- At the centerline $(r=0)$, symmetry conditions are imposed.

$$
v_{r}=0, \frac{\partial v_{z}}{\partial r}=0, \quad \omega=0, \frac{\partial T}{\partial r}=0, \quad \frac{\partial Y_{n}}{\partial r}=0 \quad \text { for } n=1, \ldots, N_{s}
$$

- At the outer zone $\left(r=r_{\max }\right)$, vanishing radial gradients are imposed for all variables except $v_{r}$.

$$
\frac{\partial v_{z}}{\partial r}=0, \frac{\partial \omega}{\partial r}=0, \frac{\partial T}{\partial r}=0, \frac{\partial Y_{n}}{\partial r}=0 \quad \text { for } n=1, \ldots, N_{s}
$$


Along the outer zone boundary, the radial velocity $v_{r}$ is obtained by solving the continuity equation.

- At the outflow $\left(z=z_{\max }\right.$ ), axial gradients of all variables (except $v_{r}$ ) are forced to vanish.

$$
\frac{\partial v_{z}}{\partial z}=0, \frac{\partial \omega}{\partial z}=0, \quad \frac{\partial T}{\partial z}=0, \frac{\partial Y_{n}}{\partial z}=0 \quad \text { for } n=1, \ldots, N_{s}
$$

Along the outflow boundary, $v_{r}$ is obtained from the continuity equation.

- At the inlet $(z=0)$, the following conditions are imposed, with the mass fractions in the fuel jet corresponding to $65 \% \mathrm{CH}_{4}$ and $35 \% \mathrm{~N}_{2}$ on a molar basis.

$$
r \leq 1:\left\{\begin{array}{l}
u_{z}=U_{0}(r, t) \\
u_{r}=0 \\
T=1 \\
Y_{\mathrm{CH}_{4}=0.515} \\
Y_{\mathrm{N}_{2}}=0.485
\end{array} \quad 1+e \leq r \leq b:\left\{\begin{array}{l}
u_{z}=U_{c} \\
u_{r}=0 \\
T=1 \\
Y_{\mathrm{O}_{2}}=0.232 \\
Y_{\mathrm{N}_{2}}=0.768
\end{array}\right.\right.
$$

Also, on the fuel tube rim $(1 \leq r \leq 1+e)$ and in the "dead zone" beyond the coflow $\left(r>b=12.5\right.$ ), no-slip conditions are set with $u_{r}=u_{z}=0$, along with $T=1, Y_{\mathrm{O}_{2}}=$ 0.232 , and $Y_{\mathrm{N}_{2}}=0.768$. Mass fractions of all species other than those specified above are set to 0 , and $\omega$ is determined from its definition in terms of $v_{r}$ and $v_{z}$.

The time variation of the flow is introduced by imposing a periodic modulation of the fuel velocity at the inlet, $U_{0}(r, t)=\left(1-r^{2}\right)(1+A \sin t)$, where the amplitude of the fluctuation is $A=0.5$ in all computations below. Boundary conditions in the air coflow correspond to injection through a porous wall as done previously in some experimental [30] and numerical [31] work. Accordingly, we assume that the coflow exits the burner with uniform velocity $U_{c}=0.5$ and that air species mass fractions are not affected by axial diffusion.

\section{Numerical method}

In order to solve the governing equations and boundary conditions numerically, the continuous domain is replaced with a structured grid composed of $N_{r} \times N_{z}$ nonuniformly distributed points, where $N_{r}=116$ and $N_{z}=162$ for the results presented here. The biggest clustering of points occurs in the region where the flame is attached, giving a minimum spacing of $\Delta z_{\min }=\Delta r_{\min }=0.025$. On this nonuniform grid, the coupled elliptic nonlinear governing equations and boundary conditions given in Equations (2) (12) are discretized via finite differences; central differences are used for first and second derivatives while upwind discretization is used for the convective terms. The unsteady terms are approximated via second-order backward differentiation as first done in [20], to avoid the convergence problems reported in [32]. The time step is adaptively chosen based on the condition that the CFL number cannot exceed a limiting value of 0.7 and typically varies between $\Delta t=0.001$ and $\Delta t=0.01$. This restriction on the CFL number is imposed so that temporal accuracy can be controlled; however, there are no limits on the CFL number coming from stability considerations, because the discretization and the solution method are fully implicit. 
Following the methodology employed in $[17,20,21,33]$, the computation uses the solution obtained for a previously computed steady flame as its initial condition and evolves the flame in time. At each time level, the values of $N_{\text {points }} \times N_{\text {dep }}$ dependent variables (namely $v_{r}, v_{z}, \omega, T$, and $Y_{n}$ with $n=1, \ldots, N_{s}$ ) must be determined, where $N_{\text {points }}=N_{r} \times$ $N_{z}$ and $N_{\text {dep }}=4+N_{s}$. To compute these values, the strongly coupled, highly nonlinear discretized equations are solved by means of a fully implicit Newton iterative method as follows. The discretized equations are rearranged in residual form $F(\Phi)=0$, where $\Phi$ is the (as yet unknown) solution vector containing all dependent variables at all grid points. The system is then solved iteratively with a damped modified Newton's method $[34,35]$, which has been used successfully in several previous flame studies such as $[17,20,21,29$, $33,36-39]$. The $k$ th iteration takes the form

$$
J\left(\Phi^{k}\right)\left(\Phi^{k+1}-\Phi^{k}\right)=-\lambda^{k} F\left(\Phi^{k}\right),
$$

with $0<\lambda^{k} \leq 1$ representing the $k$ th damping parameter [34] and $J\left(\Phi^{k}\right)=\partial F / \partial \Phi$ the Jacobian matrix evaluated at $\Phi^{k}$. At each time level, the iterative process is started with an initial guess $\Phi^{0}$ sufficiently close to the solution, and the iteration continues until the norm of the difference between two consecutive solution vectors falls below a preset tolerance. Within each iteration, the linear system in Equation (13) is solved using a Bi-CGSTAB linear solver [40] with a block-row Gauss-Seidel preconditioner. Bi-CGSTAB is considered to have converged when the 2-norm of the scaled linear system residual vector is less than one-tenth of the convergence tolerance used in Newton's method. The combination of BiCGSTAB with block-row Gauss-Seidel preconditioning, which was first developed and applied to flames in $[41,42]$, has been employed in the simulation of many two-dimensional reacting flows since 1994; for example, see [17,20,21,33,36-39, 43].

It should be noted that the solution of the system described by Equation (13) has been optimized for a predetermined sparsity structure of the Jacobian matrix, with no more than nine nonzero entries in every row or column. This fact complicates the treatment of the boundary conditions since, to ensure mass conservation, second-order-accurate discretizations must be achieved without altering the predetermined Jacobian sparsity. A detailed description of the discretization employed at the boundaries in the current work can be found in [22]; other boundary condition discretizations derived under similar constraints appear in [44].

Using the Newton-based method detailed above, the dependent variables are computed at successive time levels until the solution attains full periodicity, meaning that the initial transient features of the problem have died out. Computationally, this state is reached when the differences between the results obtained for two consecutive cycles are "small enough." To this end, we define the error $\varepsilon$ as

$$
\varepsilon=\sum_{i=1}^{N_{r}} \sum_{j=1}^{N_{z}} \frac{\left|\Phi_{i, j}(t)-\Phi_{i, j}(t+2 \pi)\right|}{\Phi_{i, j}}
$$

whose value is calculated at the end of every oscillation cycle. The computation terminates when $\varepsilon$ falls below a prescribed tolerance. 


\section{Results}

Before examining the computational results in detail, we briefly review the behavior of unforced time-dependent flames, because the existence of a natural flickering frequency will be seen to influence the computational results obtained for the forced time-dependent flames presented below. Many unforced laminar jet diffusion flames burning in oxidizing environments oscillate or flicker as a result of Kelvin-Helmholtz instabilities that appear in the buoyancy-induced shear layer surrounding the flame surface [45]. This unsteady behavior observed in low-Reynolds-number flames can be characterized by its oscillatory frequency, typically ranging from 10 to $20 \mathrm{~Hz}$ depending on the pressure level. At atmospheric pressure, experimental [46-48] and numerical [49] studies localize the natural oscillation frequency at around $12 \mathrm{~Hz}$, independent of the fuel considered or the burner size. For the flame parameters in the present work, a frequency of $12 \mathrm{~Hz}$ corresponds to a Strouhal number of $S \simeq 0.2$. However, apart from this value of the Strouhal number, to which we will refer when explaining some phenomena we have observed, we will no longer focus upon the behavior of unforced flames. In fact, our $S=0$ flame, which is essentially a nondimensionalized version of the steady flame examinined both numerically and experimentally in $[30,36,50,51]$, contains no observable or measurable oscillations.

The rest of this section examines the combustion of a nitrogen-diluted methane jet discharging into an air coflow for different fuel jet excitation frequencies. All results were computed using a 1.994-GHz Opteron Model 246 with 7.9 GB of RAM, of which each flame calculation required approximately $0.5 \mathrm{~GB}$. In the discussion below, the unsteady flames will be compared with the steady flame obtained by imposing $S=0$ in Equations (2)-(12), which from now on will be referred to as the steady flame solution. We also mention here that pinch-off of an unsteady flame is characterized by the formation of a distinct region of high-temperature reacting fluid that separates from the main wishboneshaped flame region, drastically reducing the flame height $H_{T}$. The time at which pinch-off occurs is the moment at which this separation occurs, and it is determined by checking the flame height along the oscillation cycle (see Section 4.1.4).

\subsection{Flow characterization}

\subsubsection{Temperature and concentration fields}

Isotherms are plotted in Figures $2-5$ for $S=0.1,0.25,0.5$ and 1 , respectively, while the steady solution $S=0$ is plotted in Figure 1. For small values of the Strouhal number $(S=0.1)$, the modulation of the fuel inlet velocity is so slow that the jet has enough time to adapt to the fuel mass flow modulation by modifying the temperature and species mass fraction distributions. The two flame features that display the most obvious variation are the flame height, defined as the location at which the maximum temperature is reached at the centerline, and the flame width. As the amplitude of the jet inlet velocity grows, temperature isosurfaces move radially outwards to accommodate the additional mass exiting the injector. For $S=0.1$, the mass flow oscillation is very slow and no appreciable bulges are formed, but significant variations on the flame height are observed, with increases over $100 \%$.

The situation changes for larger values of $S$. For $S=0.25$, the oscillations generate a pronounced bulge that travels downstream at a certain velocity. In Figure 3 , at $t / 2 \pi=0$, the bulge generated in the previous cycle is initially located at $z \simeq 25$ and moves toward increasing $z$, thus widening the flame. Behind it, the flame is stretched by the vortex generated in the outer cold gas, reducing the flame's transverse size and quenching the reaction. At $t / 2 \pi=0.4$, the effects of the amplitude increase begin to be noticeable again 

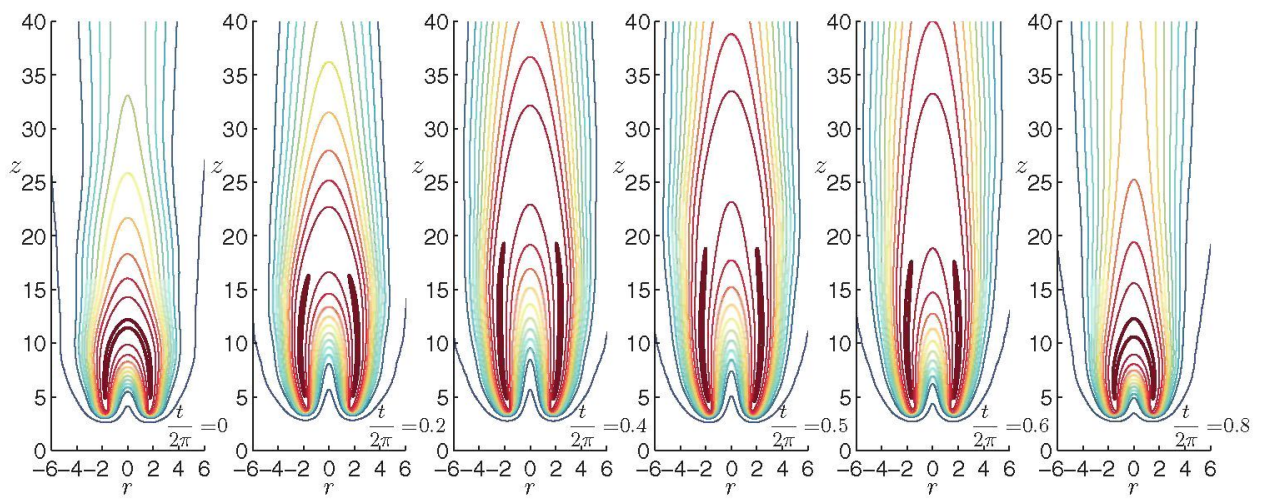

Figure 2. For $S=0.1$, isotherms are plotted at different times within a single oscillation cycle. The isotherms are equally spaced with $\Delta T=0.5$, and the thick lines indicate the maximum plotted temperature $(T=6.5)$. Note that the six plots do not occur at equispaced times.

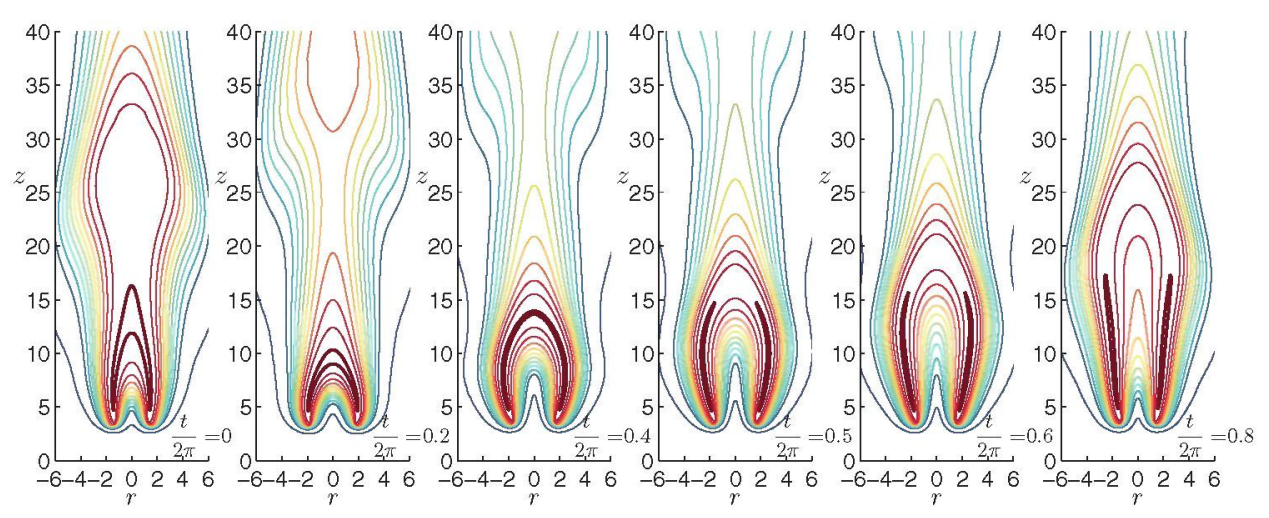

Figure 3. As for Figure 2, but with $S=0.25$.
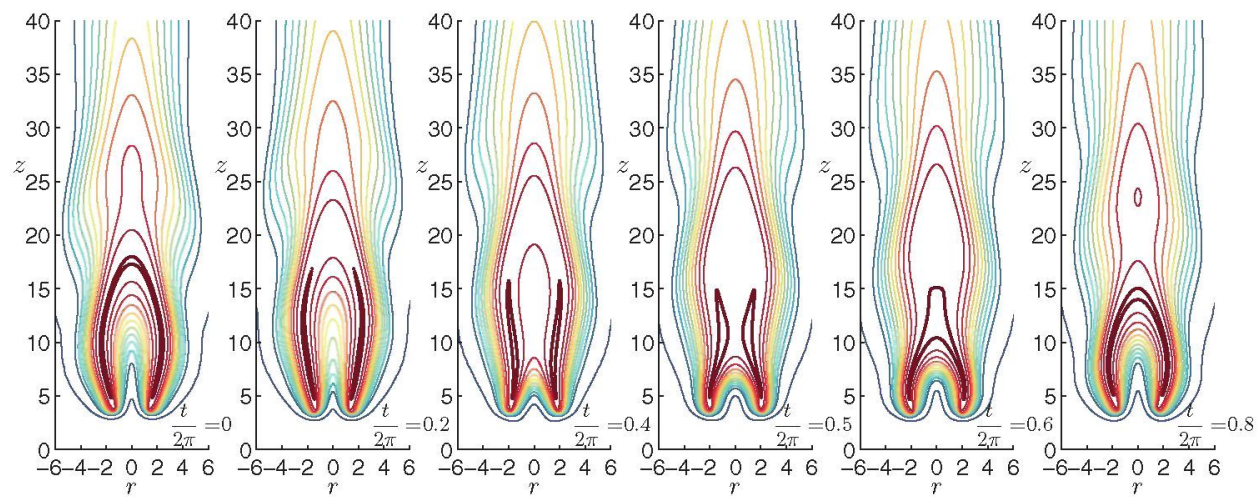

Figure 4. As for Figure 2, but with $S=0.5$. 

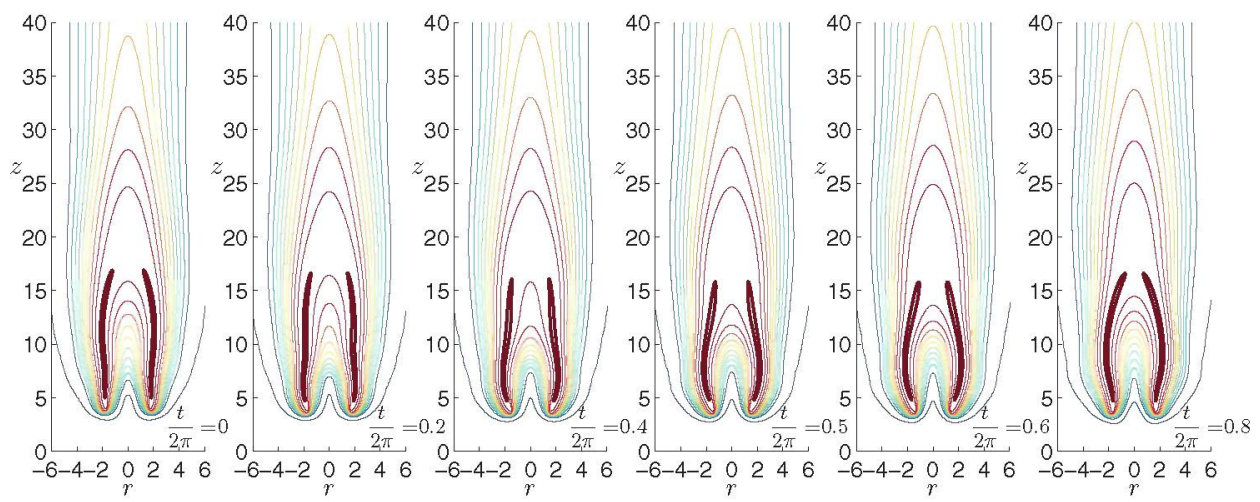

Figure 5. As for Figure 2, but with $S=1$.

at the base of the flame, with isotherms deforming as the bulge passes by and the flame length increases. After the pinch-off, which occurs around $t / 2 \pi=0.9$, the gas located in the detached hot-gas pocket rapidly cools, and the flame's height is drastically reduced between $t / 2 \pi=0.9$ and $t / 2 \pi=1$. (Recall that the results presented are fully periodic, so $t / 2 \pi=0$ is the same as $t / 2 \pi=1$.)

As the Strouhal number increases beyond 0.25 , the flame pinch-off is much less noticeable although still present, as evidenced by the succession of bulges that can be identified from the isotherms plotted in Figure 4 for $S=0.5$. The contrast between this behavior and the $S=0.25$ behavior can be explained by considering the coupling between the oscillation frequency and the unforced oscillation frequency generated by the buoyancy forces $(S \simeq 0.2$ ). Several experimental works $[6,7,52]$ have shown a similar behavior for forcing frequencies near this value, in which the primary shear instability induces the formation of vortices that lead to flame pinch-off. Another change in the thermofluid dynamics that occurs as $S$ grows is that the region affected by the oscillation on the fuel inlet velocity shrinks, thus localizing the gas affected by the pulsation to a small region located near the jet exit, similar to what happens in the classical Stokes problem of an oscillating plate. For this reason, jet pinching is only observed for computations with $S$ located in a limited range.

When the Strouhal number is even further increased to $S=1$, the flame no longer pinches off (see Figure 5) and instead takes on a character similar to the unforced flame, except that small oscillations appear at a frequency coincident with the forcing frequency. This regime has also been identified by Strawa and Cantwell [6,7], and they call it the weakly coupled state. Figure $5(S=1)$ demonstrates that the effect of the variation on the mass flow rate is reduced to the presence of small-amplitude wiggles in a region located very close to the centerline of the jet.

Methane mass fraction and the temperature along the centerline are plotted in Figures 6 and 7, respectively. The effect of the oscillation is clear from the graphic and reaffirms the results explained with Equation (1) in the Introduction. Figure 6 demonstrates that small frequencies of oscillation give rise to a perturbation that travels downstream and reaches very far distances, introducing important temporal variations of $Y_{\mathrm{CH}_{4}}$ at any given $z$ location. For $S=1$, the oscillation is reduced to small-amplitude wiggles around the steady value; these wiggles decay at almost the same rate as the steady solution. In Figure 7, temperatures slightly above the steady flame's maximum centerline temperature $T_{\max , C \text {,st }}=6.437$ are 

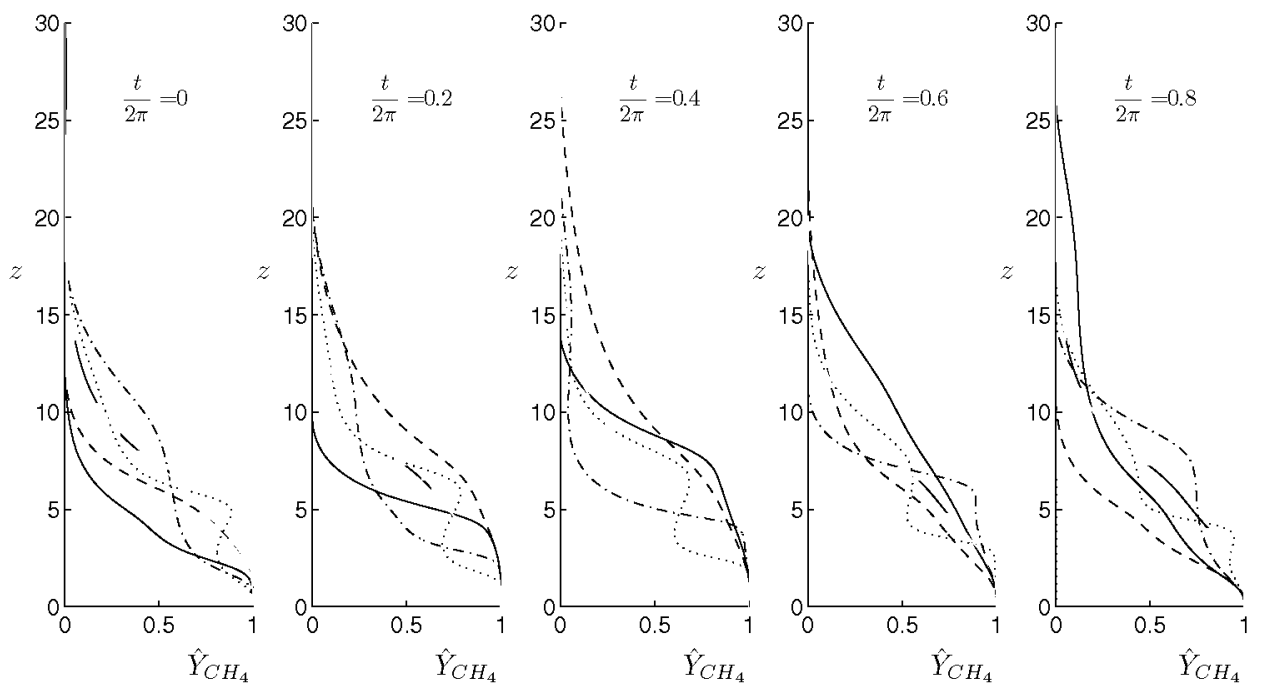

Figure 6. Methane mass fraction $\hat{Y}_{\mathrm{CH}_{4}}=Y_{\mathrm{CH}_{4}} / Y_{\mathrm{CH}_{4}, \max , C \text {, st }}$ along the centerline, with $Y_{\mathrm{CH}_{4}, \max , C \text {, st }}=$ 0.515 being the steady maximum centerline methane mass fraction. The profiles are plotted at different times $t$ (specified in the figure) for $S=0.1$ (dashed line), $S=0.25$ (solid line), $S=0.5$ (dot-dashed line), and $S=1$ (dotted line) for $A=0.5$. The gray solid line corresponds to the solution for the steady flame.

observed for all of the frequencies studied. The relevant data will be examined in more detail in Section 4.1.4.

As a result of the flame forcing, temperature and composition at a given point will change significantly during the course of an oscillation cycle. Some illustrations of this observation appear in Figure 8 for the temperature and CO mass fraction at the point $(r=0, z=10)$. For $S=0.25$, for instance, the flame tip is below $z=10$ during the first part of the cycle $(0.08 \lesssim t / 2 \pi \lesssim 0.25)$, as shown in Figure 7 . Therefore, the chemical reaction at this point is quenched, and temperature and radical mass fractions are reduced (slightly for the temperature and much more appreciably for the radical mass fractions), as seen in Figure 8 for $T$ and $\mathrm{CO}$. Only when the hot fluid coming from upstream warms the gas and reinitiates the reaction will the concentration of radicals grow again.

From the results presented above, the frequency of the forcing emerges as the most important parameter in controlling the frequency response of the flames. The unsteady perturbation decays faster as the frequency of the oscillation grows, and only for small Strouhal numbers is it possible to notice the effects of the forcing at large distances downstream. This effect has been observed previously in laminar $[6,7]$ and turbulent flames $[8,9]$.

\subsubsection{The role of viscosity}

At constant Strouhal number, it has been observed that the decay rate of the oscillatory perturbation is much faster for the combustion problem than for isothermal jets [11]. To explain this phenomenon, let us use the asymptotic expression given in Equation (1), derived recently by [12] for the decay rate of the unsteady part of the velocity in a bidimensional jet. 

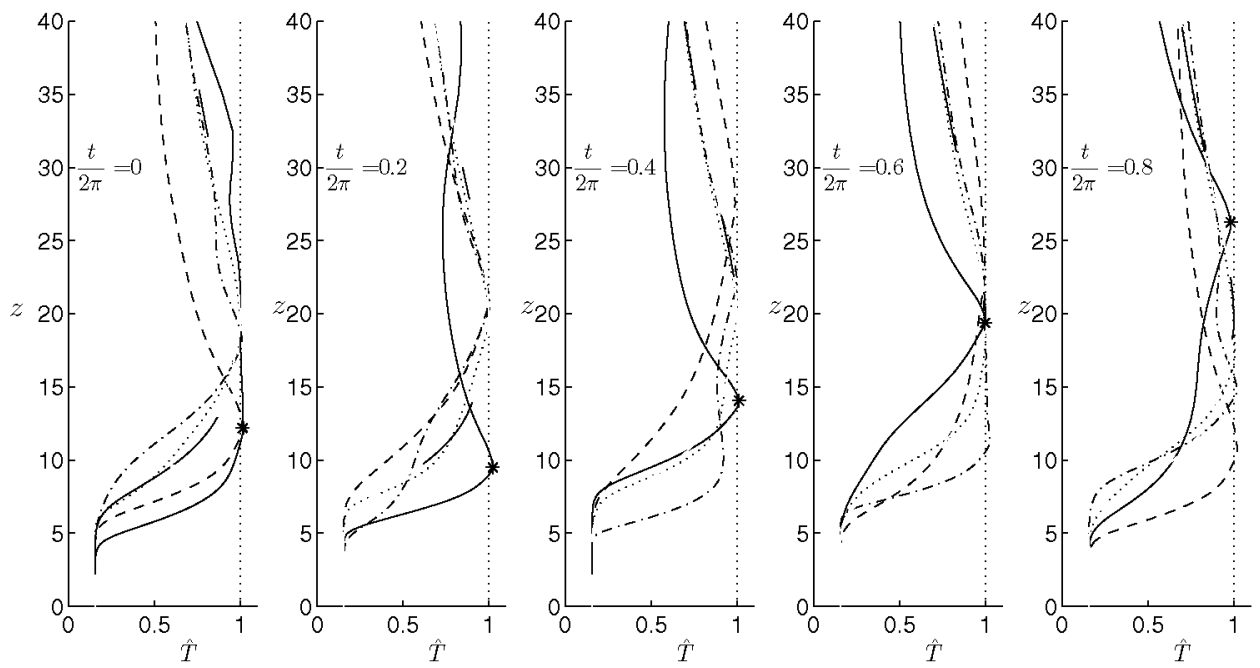

Figure 7. Temperature $\hat{T}=T / T_{\max , C \text {,st }}$ along the centerline, with $T_{\max , C, \text { st }}=6.437$ being the steady maximum centerline temperature. The profiles are plotted at different times $t$ (specified in the figure) for $S=0.1$ (dashed line), $S=0.25$ (solid line), $S=0.5$ (dot-dashed line), and $S=1$ (dotted line) for $A=0.5$. The gray solid line corresponds to the solution for the steady flame. The asterisk indicates the location of maximum temperature along the centerline for $S=0.25$.
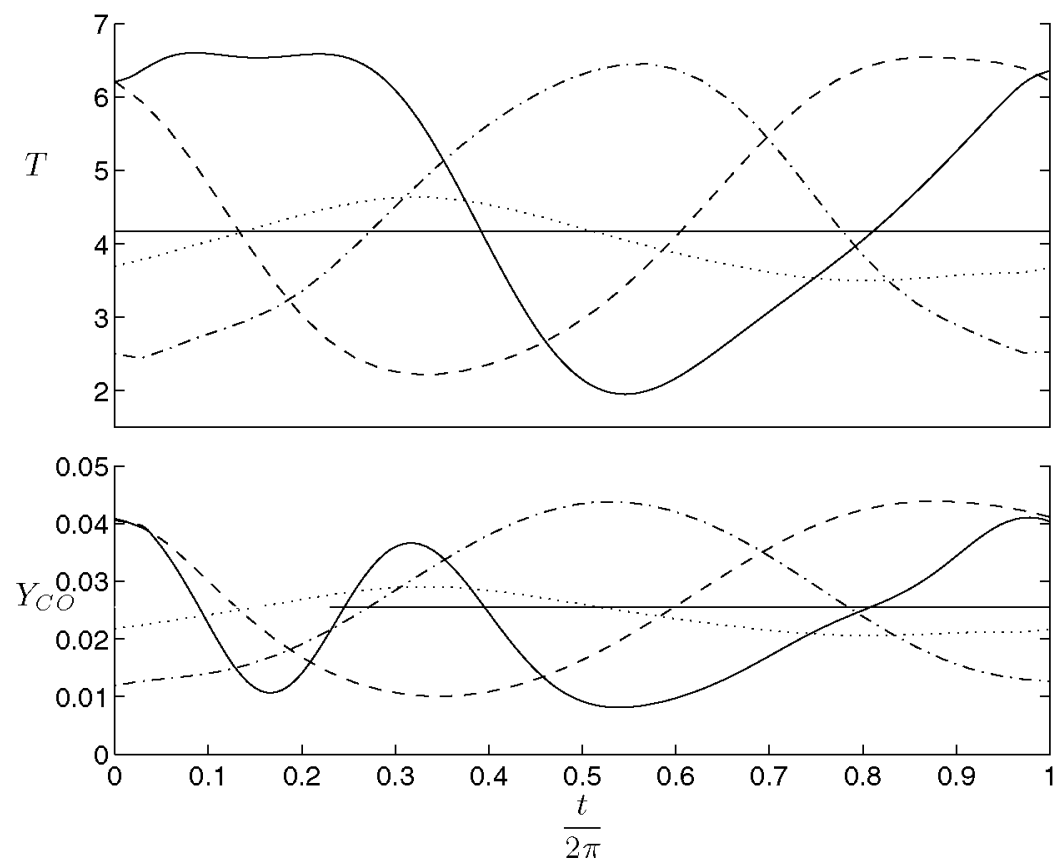

Figure 8. Temperature (upper plot) and $\mathrm{CO}$ mass fraction (lower plot) along the whole cycle at $r=0$ and $z=10$ for $S=0.1$ (dashed line), $S=0.25$ (solid line), $S=0.5$ (dot-dashed line) and $S=1$ (dotted line). The solid horizontal line in gray represents the values obtained for the steady flame $(S=0)$. 
Assuming that this expression holds for round jets, the axial distance that the perturbation can travel will be of order $z_{u} \sim\left(v^{4 / 3} S\right)^{-3 / 4}$. Therefore, as the viscosity grows due to the high temperatures achieved in combustion, $z_{u}$ will be significantly reduced. Also, because of this increase in viscosity, the flow becomes very sensitive to frequency variations.

Our results illustrate this point as follows. The flame response changes from the full modulation represented in Figure 2 for $S=0.1$ to the almost unchanged pattern of Figure 5 at a relatively small Strouhal number $S=1$; in the latter case, the oscillations do not disappear but are kept confined to the cold region located upstream of the flame. Similar results have been observed previously in the numerical solution of [10] and in the experimental works of [7-9].

\subsubsection{Periodicity}

For the four time-dependent flames, the frequency response of temperature is illustrated at a single point $(r=0, z=10)$ in Figure 9. (This position is the same one at which the same $T$ data were presented in a time-periodic way in Figure 8 , along with CO mass fraction data.) A primary motivation for Figure 9 is our intent to find the subharmonic response reported by Williams et al. in [53] for the modulation amplitudes and frequencies used in our computations. In their work, the authors used a pulsed slot burner to investigate the buoyant flame response for various fuel pulsing frequencies and amplitudes. They discovered that, under some conditions, the frequency response of the flame occurs at the pulsation frequency $w_{j}$ and its harmonics $w_{j} / n, n=1,2, \ldots$ However, as shown by Figure 9, the fast Fourier transform (FFT) of our computations did not display the presence of subharmonic responses for any of the frequencies considered, neither for the coordinate location illustrated in Figure $9(r=0, z=10)$ nor for any other coordinate locations examined throughout the domain. Apart from [53], subharmonic responses have only been reported twice $[54,55]$ and, as recognized by the authors of those works, such behavior is not present in most previous studies of modulated buoyant diffusion flames.

\subsubsection{Flame pinch-off}

Flame pinch-off is created by the presence of pairs of counter-rotating streamwise vortices [52] that stretch and quench the flame. As an illustration, three snapshots of the vorticity, temperature, and flame shape are shown in Figure 10 during the part of the cycle immediately surrounding the pinch-off phenomenon in the $S=0.25$ flame. (The flame shape is identified by the stoichiometric surface $Z=Z_{\text {stoich }}$, as explained at the beginning of Section 4.2.) For $S=0.25$, pinch-off is observed slightly after $t / 2 \pi=0.95$. As can be seen in the middle and rightmost panels of Figure 10, the coupling between the forcing frequency and the flame's natural oscillation frequency induces a vortex in the surrounding cold gas that pushes and stretches the flame, thereby quenching it and thus forming a pocket of hot gas that moves downstream.

At the instant of pinch-off, sudden changes in temperature and flame height occur. Figure 11 displays the evolution of the maximum temperature along the centerline $T_{\max , C}$ and the flame height $H_{T}$, together with the variation of the jet inlet velocity $U_{0}$ during a cycle. While this figure shows data for four flames with widely varying Strouhal numbers, an abrupt flame pinching can be observed in the $S=0.25$ and $S=0.5$ flames, in particular, at respective $t / 2 \pi$ values of approximately 0.95 and 0.5 . Immediately after pinch-offoccurs, these two flames shrink below the steady flame height $H_{T \text {,st }}=19.690$, and their maximum temperatures grow to values above the maximum steady flame temperature $T_{\max , C \text {,st }}=$ 

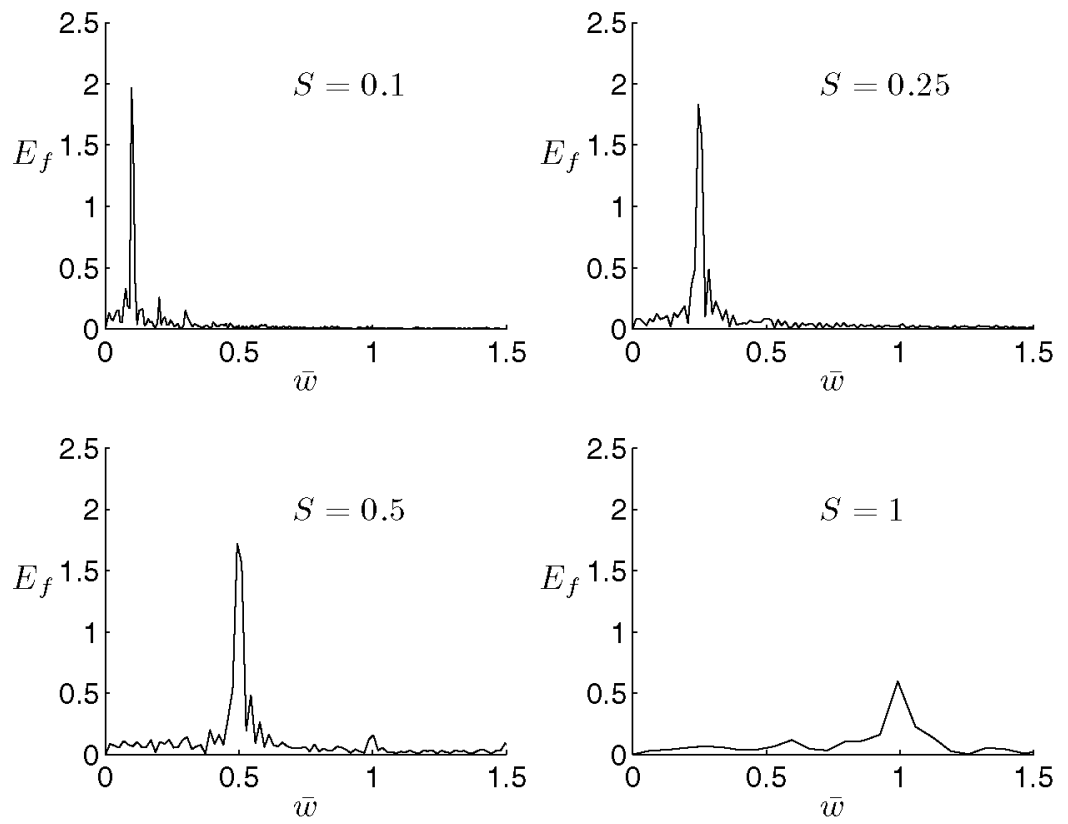

Figure 9. Fast Fourier transform (FFT) of the time-dependent temperature data at $r=0$ and $z=$ 10 for different values of Strouhal number $S$. In the plots above, $E_{f}(\bar{w})=\sum_{n=1}^{N} T(r=0, z=$ $\left.10, t_{n}\right) e^{-i \bar{w} t_{n}}$ represents the nondimensional energy contained at each frequency $\bar{w}$, and $t_{n}$ is the time defined as in Section 2.

6.437. Finally, time-averaging the $H_{T}$ data demonstrates that the average flame heights of these forced flames are slightly shorter than their steady counterpart, fundamentally due to the enhanced mixing promoted by the oscillation. This phenomenon has also been observed experimentally $[7,8]$ and numerically $[21]$.

\subsection{Flame identification}

The definition of the flame surface in a detailed-chemistry calculation is not straightforward since the finite burning rate gives the flame a certain thickness. Some authors identify the flame location with the regions of higher heat release [56] or with the hydrogen concentration [26]. Traditionally, in the study of diffusion flames with Lewis number of all species equal to unity, the mixture fraction $Z$ is the parameter used to describe the flame, with $Z$ defined as

$$
Z=\frac{S_{m_{\mathrm{F}}} Y_{\mathrm{F}} / Y_{\mathrm{F}_{0}}+1-Y_{\mathrm{O}} / Y_{\mathrm{O}_{0}}}{1+S_{m_{\mathrm{F}}}} .
$$

Here, $S_{m_{\mathrm{F}}}$ is the mass of air needed to burn all of the fuel under stoichiometric conditions, and $Y_{\mathrm{F}_{0}}=0.515$ and $Y_{\mathrm{O}_{0}}=0.232$ are the initial mass fractions of methane and air, respectively. The overall reaction for the combustion of $\mathrm{CH}_{4}$ in air is given by

$$
\mathrm{CH}_{4}+2 \mathrm{O}_{2} \rightleftharpoons \mathrm{CO}_{2}+2 \mathrm{H}_{2} \mathrm{O}
$$



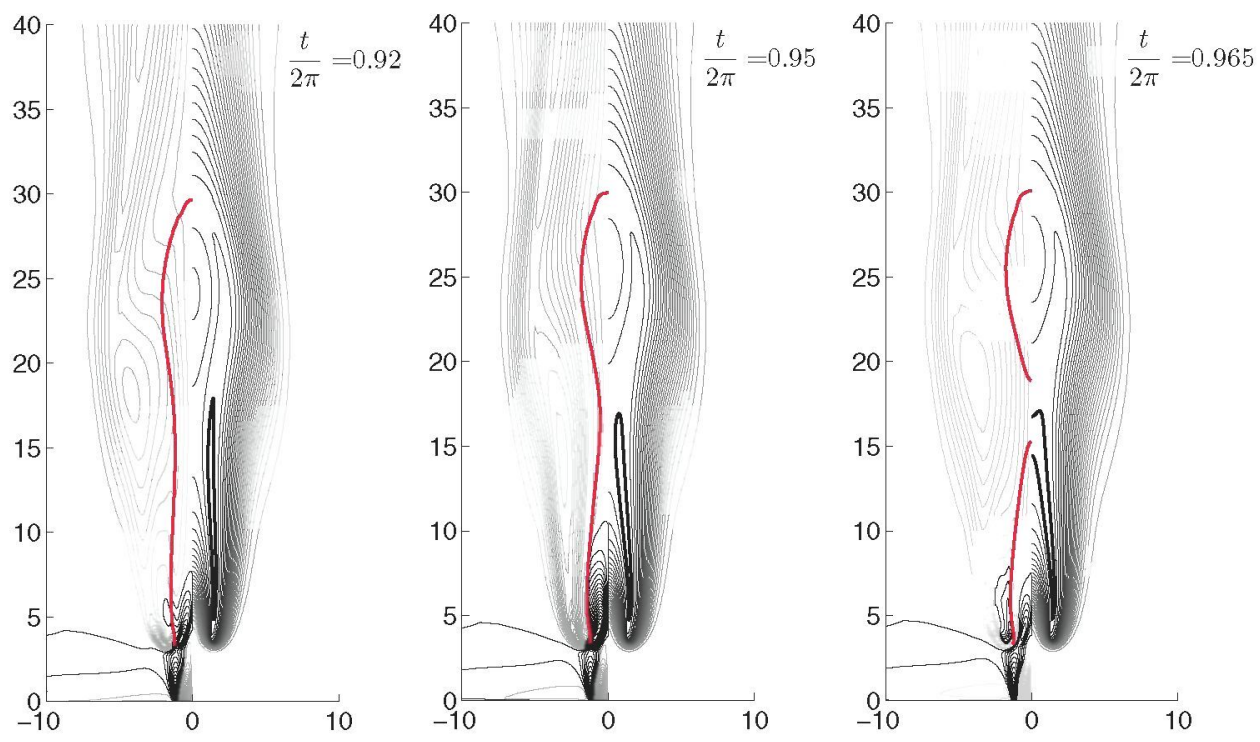

Figure 10. Vorticity and flame shape (left half of the plots) and temperature (right half of the plots) for the unsteady flame with $S=0.25$. Vorticity isopleths are plotted with $\Delta \omega=0.05$ from -5 to 5 . Positive vorticity is in gray while negative vorticity is in black. The flame shape (thick line in the left half of the plots) has been identified with the surface $Z=Z_{\text {stoich }}$, as explained at the beginning of Section 4.2. Isotherms are plotted with $\Delta T=0.2$ from $T=1.1$ to $T=6.5$. The lines darken as the temperature grows. The thick line (right half of the plots) represents the maximum plotted temperature $T=6.5$.
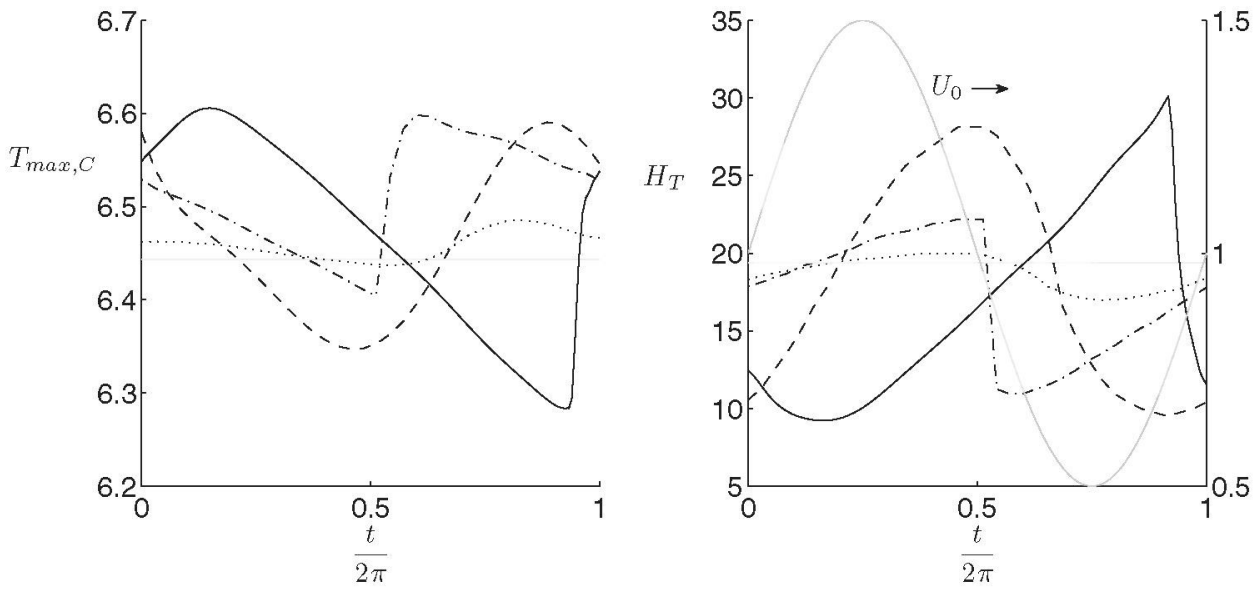

Figure 11. Variation of the maximum temperature at the centerline $T_{\max , C}$ (left plot) and flame height $H_{T}$ (left vertical axis in the right plot) with time $t$ for $S=0.1$ (dashed line), $S=0.25$ (solid line), $S=0.5$ (dot-dashed line), and $S=1$ dotted line) and amplitude $A=0.5$. In the right plot is included the variation of the jet inlet velocity $U_{0}$ (right vertical axis) with $t$. The solid horizontal lines in gray represent the values obtained for the steady flame. 
with $S_{m_{\mathrm{F}}}=8.877$. In the infinitely-fast chemistry approximation, the flame reduces to a layer of zero thickness in which $Z=Z_{\text {stoich }}=1 /\left(1+S_{m_{\mathrm{F}}}\right)$. As stated by Williams [27], this definition is appropriate far from extinction and when the Lewis number of all species is one. In our case, since the Lewis numbers of $\mathrm{CH}_{4}$ and $\mathrm{O}_{2}$ are each close to unity, we can use the previous definition to identify the flame location. Through this procedure, the number of points used to describe the flame, denoted by $N_{p}$, is 67, and the total arclength $s_{F}$ along the flame can be defined as

$$
s_{F}=\sum_{i=2}^{N_{p}} \sqrt{\left(r_{F_{i}}-r_{F_{i-1}}\right)^{2}+\left(z_{F_{i}}-z_{F_{i-1}}\right)^{2}} .
$$

The arclength parameter $s$ runs from $s=0$, which is located at $(r, z)=\left(0, H_{T}\right)$, to $s=s_{F}$, through all points $\left(r_{F}, z_{F}\right)$ at which $Z=Z_{\text {stoich }}$.

For the four time-dependent flames, flame position is plotted in the left half of Figure 12 at various times in the cycle, and the effect of the pulsation is clearly seen. While the frequency of oscillation is small, the flame keeps its initial shape but changes its length and, only slightly, its location. As $S$ grows, the shape of the flame changes significantly, folding the flame surface and eventually breaking it because of quenching motivated by large strain rates. In the insets of the plots corresponding to $S=0.25$ and $S=0.5$ in Figure 12, we show the flame shape and temperature evolution just before and after the breaking of the flame surface. For even larger Strouhal numbers, the flame deformation is still present but its effects are reduced to small wiggles that do not provoke extinction, and they do not present large departures from the steady flame shape.

The right half of Figure 12 shows the variation of the flame temperature with respect to arclength parameter $s$. The flame temperature varies between $2 \%$ and $6 \%$ with respect to the maximum flame temperature along its length. The relatively large value of the stoichiometric air-to-fuel mass ratio $S_{m_{\mathrm{F}}}$ for methane indicates that the flame will be located relatively far from the centerline, where the effect of the pulsation is small. Therefore, the flame lift-off height $L_{f}$, defined as the smallest $z$ value for which the temperature is greater than $1000 \mathrm{~K}$, oscillates vertically only slightly, between 3.5 and 3.6. The lift-off height reported experimentally in [21] for a completely analogous configuration, with Strouhal number $S=0.36$ and jet radius $a=0.2 \mathrm{~cm}$, is $0.685 \mathrm{~cm}$. This value corresponds to 3.425 in our nondimensional notation, illustrating excellent agreement between the numerics of the present study and the experiments in [21]. In our computations, the $r$ coordinate of the lowest point at which $T$ exceeds $1000 \mathrm{~K}$ varies between $\left[\begin{array}{lllll}1.401 & 1.296 & 1.341 & 1.446\end{array}\right]$ and [ 1.8791 .8771 .9761 .856 f for $S=0.1,0.25,0.5$, and 1 , respectively; these radial variations are considerably greater than the axial variations in $L_{f}$.

Despite the strong forcing, the maximum flame temperature does not change with respect to the steady problem $\left(T_{\max , s t}=6.604\right)$ and is only slightly smaller than the adiabatic flame temperature $\left(T_{\text {adiabatic }}=6.889\right)$ corresponding to the composition given in Equation (12). However, its location $\left(r_{F_{\max }}, z_{F_{\max }}\right)$ does change appreciably, oscillating around the maximum temperature location of the steady flame, as indicated by the empty dots of Figure 12. For $S=0.1$ and $S=0.25$, the variations on the mass flow rate create large transverse oscillations (as seen by the large changes in $r_{F_{\max }}$ ), while $z_{F_{\max }}$ remains almost constant. As the frequency of the pulsation grows $(S=0.5)$ compared to the variations in the mass flow rate, the flame temperature also changes due to the large strain rate generated by the pulsation, modifying the location in both the radial and axial directions. The combination of both effects results in the movement of the $\left(r_{F_{\max }}, z_{F_{\max }}\right)$ point in a loop 

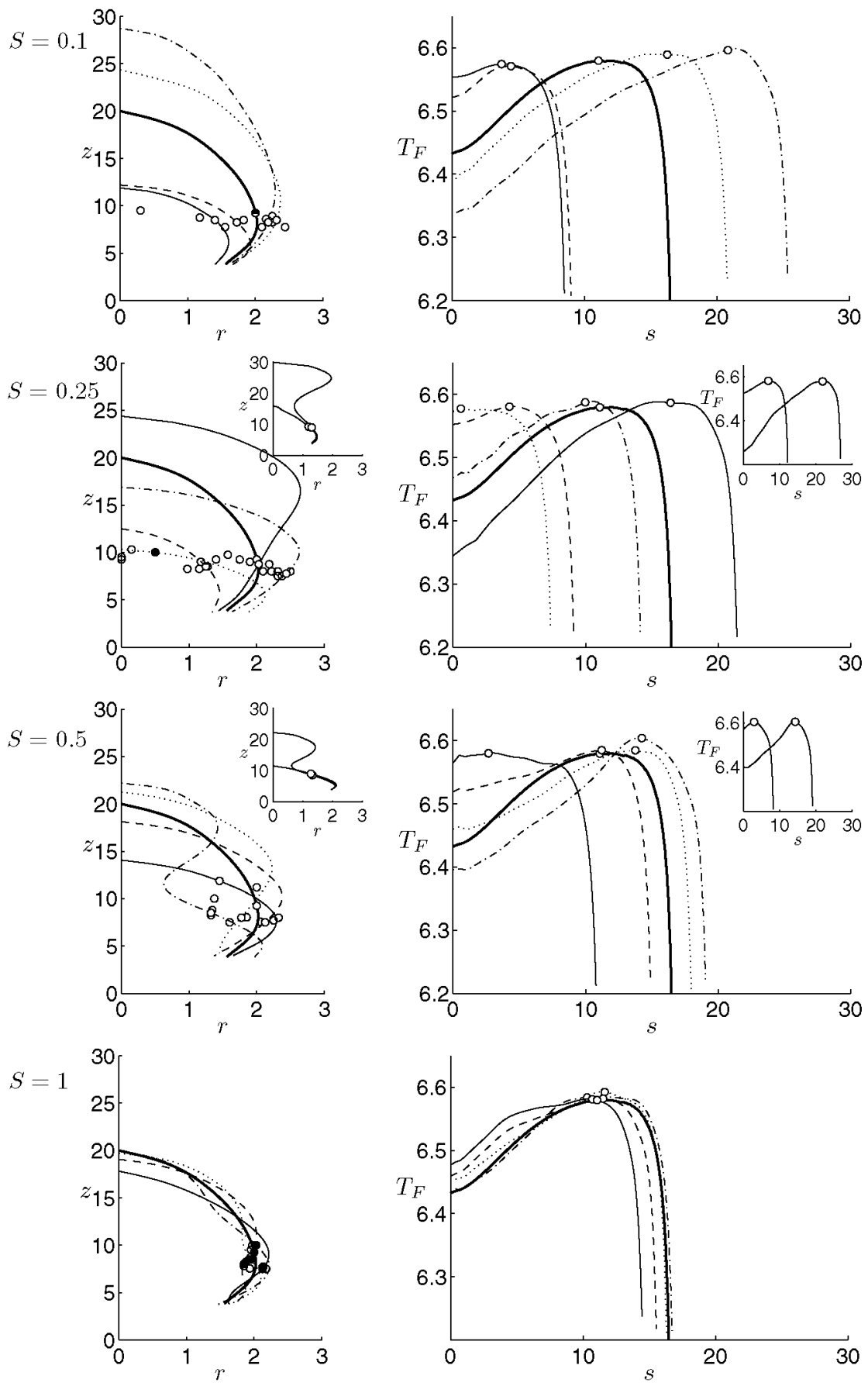

Figure 12. Flame position (left plot) and temperature (right plot) along the flame's arclength $s$ at $t / 2 \pi=0$ (dashed line), $t / 2 \pi=0.25$ (dotted line), $t / 2 \pi=0.5$ (dot-dashed line), and $t / 2 \pi=0.75$ (solid line). The thick solid line represents the solution for the steady problem. The symbols $\circ$, which represent maximum flame temperature location for every time $t$ computed, are filled at $t / 2 \pi=$ $0,0.25,0.5,1$. The insets in the plots corresponding to $S=0.25$ and $S=0.5$ show the flame shape and temperature just before and after pinch-off. 


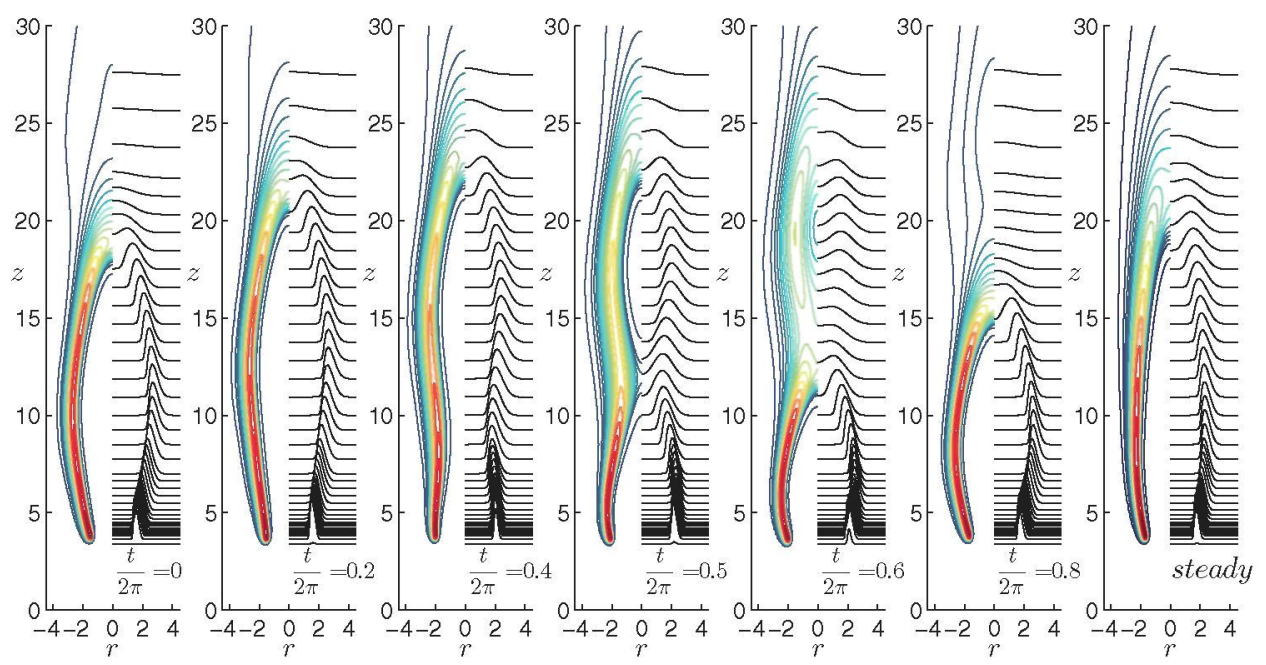

Figure 13. Normalized $\mathrm{OH}$ mass fraction $\hat{Y}_{\mathrm{OH}}=Y_{\mathrm{OH}} / Y_{\mathrm{OH}, \max , \text { st }}$ (left half of plots) at different times $t$ for $S=0.5$ compared with the steady solution (rightmost panel). Equally spaced isopleths with $\Delta \hat{Y}_{\mathrm{OH}}=0.1$ are shown for $\hat{Y}_{\mathrm{OH}} \in\left[\begin{array}{ll}0 & 1\end{array}\right]$. The color of the lines progresses from blue to red as $\hat{Y}_{\mathrm{OH}}$ grows. Right half of the plots shows $2 \times \hat{Y}_{\mathrm{OH}}$ transverse profiles at selected $z . Y_{\mathrm{OH} \text {,max, st }}=0.0032$ is the maximum $\mathrm{OH}$ mass fraction for the steady flame.

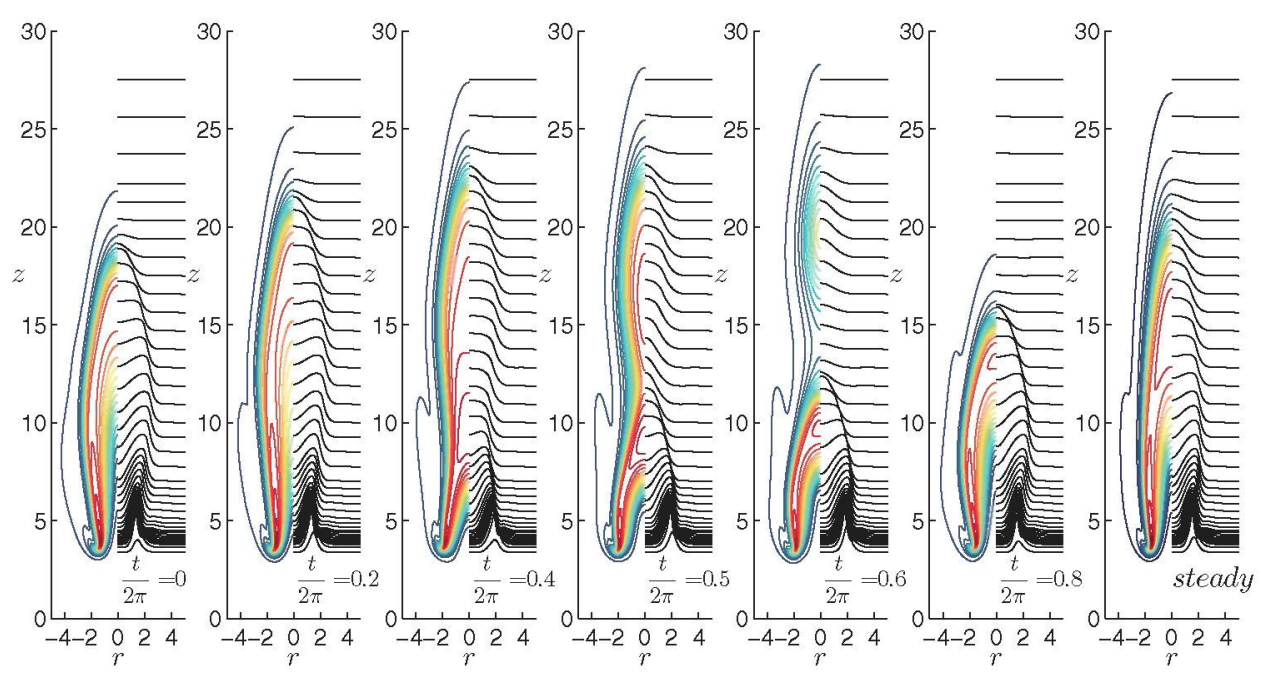

Figure 14. Normalized CO mass fraction $\hat{Y}_{\mathrm{CO}}=Y_{\mathrm{CO}} / Y_{\mathrm{CO} \text {,max,st }}$ at different times $t$ for $S=0.5$ compared with the steady solution (rightmost panel). Equally spaced isopleths with $\Delta \hat{Y}_{\mathrm{CO}}=0.05$ are shown for $\hat{Y}_{\mathrm{CO}} \in\left[\begin{array}{ll}0 & 1\end{array}\right]$. The color of the lines progresses from blue to red as $\hat{Y}_{\mathrm{CO}}$ grows. Right half of the plots shows $2 \times \hat{Y}_{\mathrm{CO}}$ transverse profiles at selected $z . Y_{\mathrm{CO} \text {, max,st }}=0.044$ is the maximum CO mass fraction for the steady flame. 


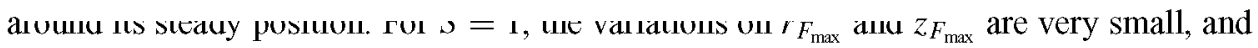
the points cluster tightly around the steady value.

\subsection{CO oxidation chemistry}

The simplest radicals $\mathrm{H}, \mathrm{O}$, and $\mathrm{OH}$ are essential intermediates in most aspects of combustion chemistry as, for example, in the formation of hydrocarbon free radicals or soot formation. Experimental measurement of $\mathrm{OH}[57]$ is the most frequently quantified radical. For a forced methane - air diffusion flame on a Wolfhard-Parker burner, Williams et al. [53] observed an appreciable thickening of the $\mathrm{OH}$ layers in their experiments. Figure 13 shows normalized $\mathrm{OH}$ mass fraction $\hat{Y}_{\mathrm{OH}}=Y_{\mathrm{OH}} / Y_{\mathrm{OH} \text {,max, st }}$ isopleths for the $S=0.5$ flame, with $Y_{\mathrm{OH}, \max , \mathrm{st}}=0.0032$ representing the maximum $\mathrm{OH}$ mass fraction attained for the steady flame. In the same figure, $\hat{Y}_{\mathrm{OH}}$ profiles at selected $z$ locations have been plotted. Confirming the experimental observations, it can be noticed that the thickness of the $\mathrm{OH}$ layer increases after the pinching at $t / 2 \pi=0.5$, an effect that has been attributed to compressive strain effects by Everest et al. [58].

The $\mathrm{OH}$ radical plays a very important role in the $\mathrm{CO}$ oxidation mechanism. The oxidation of $\mathrm{CO}$ to $\mathrm{CO}_{2}$ takes place primarily via the reaction

$$
\mathrm{CO}+\mathrm{OH} \rightleftharpoons \mathrm{CO}_{2}+\mathrm{H}
$$

where the reverse reaction is also important. Its reaction rate depends on the $\mathrm{OH}$ concentration, which the jet forcing modifies significantly as seen in Figure 13 . We note a $40 \%$ increase in the peak $\mathrm{OH}$ mass fraction along the centerline for $S=0.5$ and $S=0.1$, while maximum $Y_{\mathrm{OH}}$ values (throughout the domain) show changes of less than $2 \%$. Similar behavior has been observed for CO (Figure 14), with peak centerline variations of around $12 \%$ and small changes in the maximum values (throughout the domain) of around $3 \%$, confirming the results of previous experimental studies of flickering flames $[16,58]$ and computations of forced methane flames [21].

To quantify the total mass variation of the $n$th species, we compute

$$
m_{n}(t)=\oint_{V} Y_{n} \rho d V
$$

in the volume $V=\pi r_{\max }^{2} z_{\max }$ at each time $t$. The variation of total OH mass in a cycle is plotted in Figure 15, along with other important species in the $\mathrm{CO}$ to $\mathrm{CO}_{2}$ oxidation process, such as $\mathrm{H}, \mathrm{OH}$, and $\mathrm{CH}_{3}$. Total mass variation of the species decreases as the Strouhal number increases, as expected from previous results. Of particular note is the counterphase variation of $\mathrm{CO}_{2}$ with respect to the other species. As has been suggested previously [21], an increase in the presence of $\mathrm{H}$ and hydrocarbon radicals, such as $\mathrm{CH}_{3}$, affects the amount of $\mathrm{OH}$ that can react with $\mathrm{CO}$ in reaction (II). Alternative reactions with hydrocarbon species with much higher reaction rates consume most of the available $\mathrm{OH}$, reducing the oxidation of carbon monoxide, and therefore causing an augmentation of $\mathrm{CO}$ and a $\mathrm{CO}_{2}$ mass dropoff during the first half of the cycle. The situation is fully reversed during the second part of the cycle, in which $H$ and hydrocarbon species production has been retained.

To understand this behavior better, note that in the 15-species, 42-reaction mechanism used in the computations, fuel break-up occurs mainly through the following 

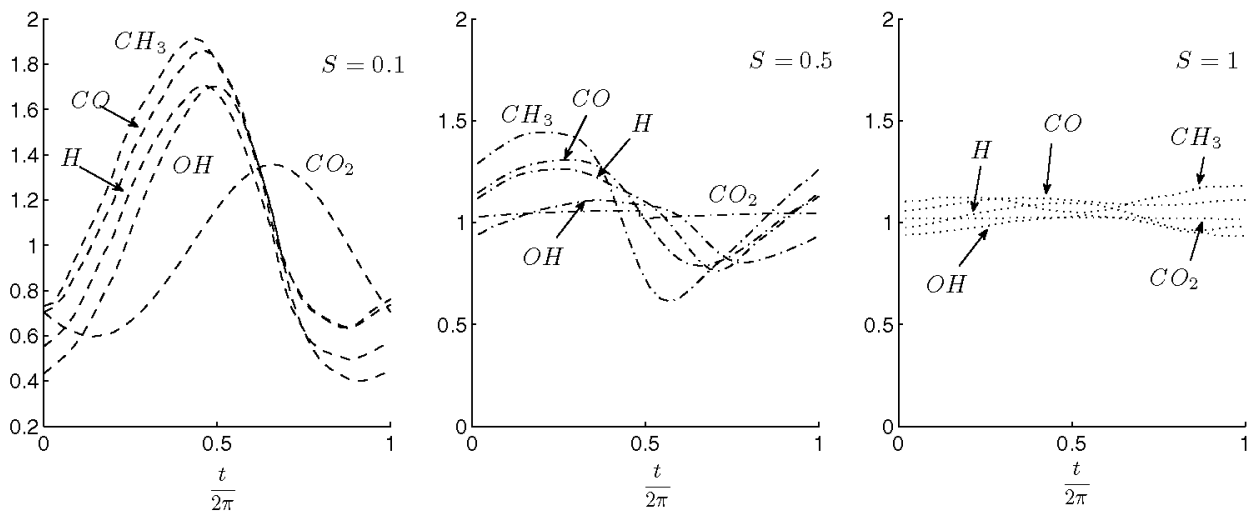

Figure 15. Scaled total mass $\hat{m}=m_{n} / m_{n, \max , \text { st }}$ for different species throughout an oscillation cycle, with $m_{n \text {,max,st }}=2 \pi \times\left[1.33 \times 10^{-4}, 0.011,0.152,8.409,0.024\right]$ for $\mathrm{H}, \mathrm{OH}, \mathrm{CO}, \mathrm{CO}_{2}$, and $\mathrm{CH}_{3}$, respectively.

reactions $[59,60]$ :

$$
\begin{aligned}
\mathrm{CH}_{4}+\mathrm{H} & \rightarrow \mathrm{CH}_{3}+\mathrm{H}_{2} \\
\mathrm{CH}_{4}+\mathrm{OH} & \rightarrow \mathrm{CH}_{3}+\mathrm{H}_{2} \mathrm{O} \\
\mathrm{CH}_{4}+\mathrm{O} & \rightarrow \mathrm{CH}_{3}+\mathrm{OH},
\end{aligned}
$$

where the reaction with $\mathrm{O}$ is less important than the reactions with $\mathrm{H}$ and $\mathrm{OH}$. The $\mathrm{CH}_{3}$ formed reacts to form formaldehyde $\left(\mathrm{CH}_{2} \mathrm{O}\right)$ via the reaction

$$
\mathrm{CH}_{3}+\mathrm{O} \rightarrow \mathrm{CH}_{2} \mathrm{O}+\mathrm{H}
$$

The combination of the formaldehyde with $\mathrm{H}$ and $\mathrm{OH}$ will produce $\mathrm{H}_{2}, \mathrm{OH}$, and $\mathrm{CHO}$ by the following reactions:

$$
\begin{gathered}
\mathrm{CH}_{2} \mathrm{O}+\mathrm{H} \rightarrow \mathrm{CHO}+\mathrm{H}_{2} \\
\mathrm{CH}_{2} \mathrm{O}+\mathrm{OH} \rightarrow \mathrm{CHO}+\mathrm{H}_{2} \mathrm{O} .
\end{gathered}
$$

The CHO is then converted to CO mainly by

$$
\begin{gathered}
\mathrm{CHO}+\mathrm{H} \rightarrow \mathrm{CO}+\mathrm{H}_{2} \\
\mathrm{CHO}+\mathrm{M} \rightarrow \mathrm{CO}+\mathrm{H}+\mathrm{M} \\
\mathrm{CHO}+\mathrm{O}_{2} \rightarrow \mathrm{CO}+\mathrm{HO}_{2} \\
\mathrm{CHO}+\mathrm{OH} \rightarrow \mathrm{CO}+\mathrm{H}_{2} \mathrm{O},
\end{gathered}
$$

with reaction $(\mathrm{X})$ dominating at high temperatures. The oxygen consumption and radical formation is attributed to

$$
\mathrm{H}+\mathrm{O}_{2} \rightleftharpoons \mathrm{OH}+\mathrm{O}
$$



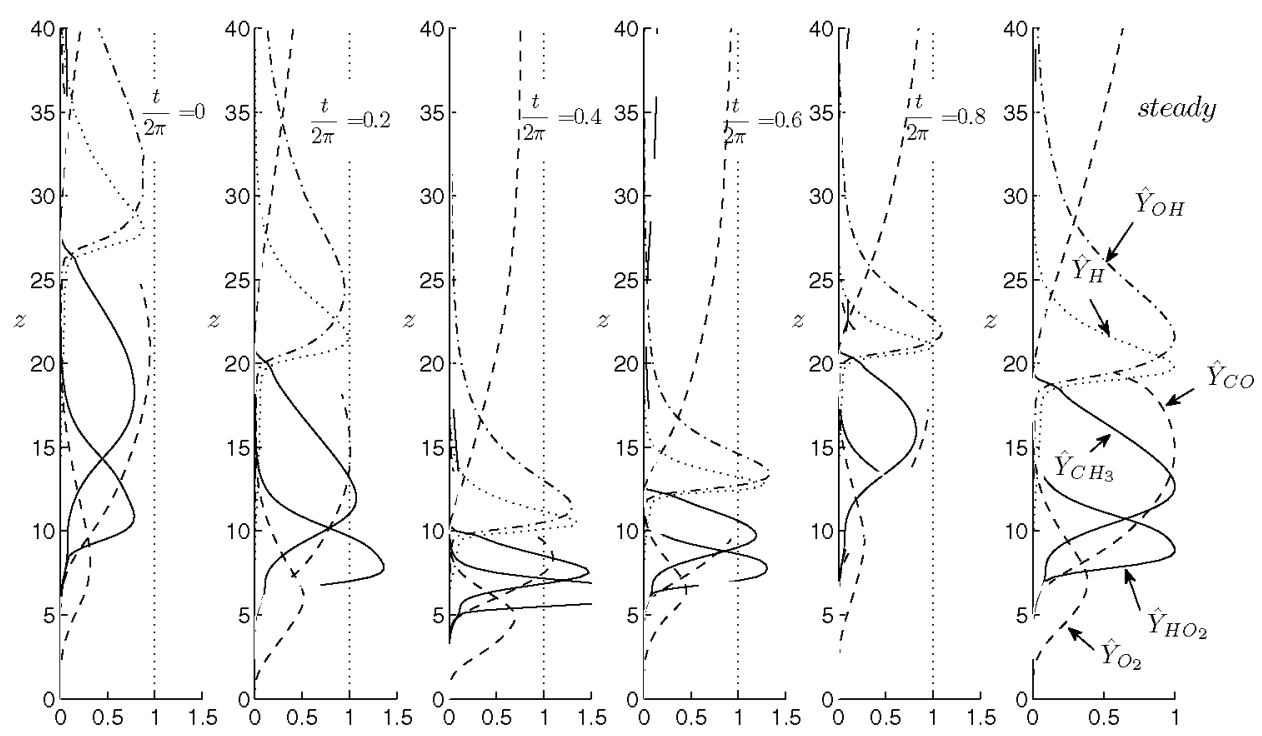

Figure 16. Scaled mass fractions $\hat{Y}=Y_{n} / Y_{n, \max , C, \text { st }}$ at the centerline, throughout an oscillation cycle for $S=0.1$. The steady solution is plotted in the rightmost panel, with a slightly stretched horizontal axis.

which is important only at high temperatures due to its high activation energy. Finally, radical recombination converts the $\mathrm{HO}_{2}$ created through

$$
\mathrm{H}+\mathrm{O}_{2}+\mathrm{M} \rightarrow \mathrm{HO}_{2}+\mathrm{M}
$$

into stable molecules via

$$
\begin{aligned}
\mathrm{H}+\mathrm{HO}_{2}+\mathrm{M} & \rightarrow \mathrm{H}_{2}+\mathrm{O}_{2} \\
\mathrm{OH}+\mathrm{HO}_{2} & \rightarrow \mathrm{H}_{2} \mathrm{O}+\mathrm{O}_{2} .
\end{aligned}
$$

In Figure 16, we plot the scaled mass fraction $\hat{Y}_{n}=Y_{n} / Y_{n, \text { max }, C \text {,st }}$ for $S=0.1$, with $Y_{n, \max , C, \text { st }}=\left[0.0012,2.68 \times 10^{-5}, 1.04 \times 10^{-5}, 0.1543,8.91 \times 10^{-4}, 0.0379\right]$ being the maximum values along the centerline obtained for $\mathrm{OH}, \mathrm{H}, \mathrm{HO}_{2}, \mathrm{O}_{2}, \mathrm{CH}_{3}$, and $\mathrm{CO}$, respectively, in the steady problem. From this figure, it is easy to see the strong relation between the available $\mathrm{O}_{2}$ and the $\mathrm{H}, \mathrm{CH}_{3}$, and $\mathrm{OH}$ radical mass fractions. During the first part of the cycle, the $\mathrm{O}_{2}$ availability is small due to small entrainment rates, and the formation of radicals through reaction (XIII) is therefore not promoted. The situation changes as the pulsation induces a larger $\mathrm{O}_{2}$ concentration available for reaction (XIII) due to the enhanced entrainment from the surrounding coflow (see Figure 16). Additional amounts of $\mathrm{OH}$ and $\mathrm{O}$ promote the formation of $\mathrm{CH}_{3}$ and $\mathrm{H}$ via reactions (III), (V), and (VI). It is only after all the $\mathrm{CH}_{\mathrm{n}} \mathrm{O}$ species have been consumed that the slow reaction (II) becomes important and most of the $\mathrm{CO}_{2}$ is formed. 


\section{Conclusion}

We have presented numerical solutions of four forced, axisymmetric, laminar, methane jet diffusion flames obtained from the integration of the full unsteady Navier-Stokes equations with a detailed chemistry scheme consisting of 15 chemical species and 42 reaction steps. In each flame, the modulation of the fuel jet velocity produces a controllable periodic flow whose characteristics depend upon the nondimensional frequency of oscillation denoted by $S$, the Strouhal number. According to our results, the frequency of the oscillation is the most important parameter in forcing jet flames. The effects of the pulsation decay faster as the Strouhal number of the flow grows, and only low frequency forcing could be detected at large distances downstream - a conclusion that has previously been experimentally confirmed for both laminar [6,7] and turbulent flames [8,9].

The flames are very sensitive to the oscillation frequency, much more so than the corresponding cold flow problem. Equivalent frequency variations lead to much sharper changes in flame behavior which, based on previous numerical calculations $[10,11]$ and on Equation (1) derived by [12], could be attributed to the strong viscosity variations induced by the combustion. A similar conclusion can also be found in the experimental work of Strawa and Cantwell [7]. For small Strouhal numbers, the flow is affected greatly by changes in the oscillation frequency, which alter the chemical dynamics such that the temperature and species concentrations are changed. As the frequency approaches the natural flickering frequency, the flame breaks up due to flame pinch-off, which is produced by the stretching induced by the vortices created in the outer cold gas. As a result, a pocket of hot gas is separated from the flame and travels downstream at constant velocity until it is cooled by the surrounding air. Larger oscillation frequencies (near $S=1$ ) lead to a regime in which the oscillations are confined to the jet's cold near-exit region. The flame remains almost unaltered, and departures with respect to the steady flame for $S=0$ become smaller as the Strouhal number grows.

The effect of the oscillation on CO oxidation chemistry has also been studied. According to the experimental studies in $[16,58]$, unsteady flames produce larger quantities of $\mathrm{CO}$ than their steady counterparts. Our computations reproduce this behavior, and the results suggest that the larger $\mathrm{O}_{2}$ entrainment rates induced by the oscillation lead to the formation of additional radicals that inhibit the oxidation of $\mathrm{CO}$.

The strong forcing introduced by a modulation of amplitude $A=0.5$ changes the shape and the maximum temperature achieved by the flame. In spite of this behavior, the lift-off height remains practically unaltered. The underlying reasons for this phenomenon are still under investigation and will be the subject of future work.

\section{Acknowledgements}

The authors gratefully acknowledge financial support from the following sources: the Spanish Ministry of Science and Innovation through the José Castillejo Mobility Program for Young Researchers (MSS) and Project \#ENE 2005-09-190-C04-01 (AL); the US Department of Energy Office of Basic Energy Sciences under Grant \#DE-FG02-88ER13966 (MDS and BAVB); the US National Science Foundation under Grant \#CBET-0828802 (MDS and BAVB); and the US Air Force Office of Scientific Research under Grant \#FA9550-06-1-0018 (MDS and BAVB). The authors also gratefully acknowledge helpful conversations with Forman A. Williams.

This work is dedicated to the memory of Dulce N. Morillo-Martín.

\section{References}

[1] D.S. Chamberlin and A. Rose, The flicker of luminous flames, Ind. Eng. Chem. 20 (1928), pp. 1013-1016. 
[2] A. Ballantyne and K.N.C. Bray, Investigations into the structure of jet diffusion flames, Proc. Combust. Inst. 16 (1977), pp. 777-787.

[3] H.A. Beker and D. Liang, Soot emission, thermal radiation and laminar instabilities of acetylene diffusion flames, Combust. Flame 52 (1983), pp. 247-256.

[4] A.J. Grant and J.M. Jones, Low frequency diffusion flame oscillations, Combust. Flame 25 (1975), pp. 153-160.

[5] A.E. Perry and M.S. Chong, A description of eddying motions and flow patterns using criticalpoint concepts, Ann. Rev. Fluid Mech. 19 (1987), pp. 125-155.

[6] A.W. Strawa and B.J. Cantwell, Visualization of the structure of a pulsed methane-air diffusion flame, Phys. Fluids 28 (1985), pp. 2317-2320.

[7] A.W. Strawa and B.J. Cantwel1, Investigation of an excited jet diffusion flame at elevated pressure, J. Fluid Mech. 200 (1989), pp. 309-336.

[8] J.A. Lovett and S.R. Turns, Experiments on axisymmetrically pulsed turbulent jet flames, AIAA J. 28 (1990), pp. 38-46.

[9] J.A. Lovett and S.R. Turns, The structure of pulsed turbulent nonpremixed jets, Combust. Sci. Tech. 94 (1993) pp. 193-217.

[10] X. Jiang, H. Zhao and L. Cao, Direct computation of a heated axisymmetric pulsating jet, Numer. Heat Transfer, Part A 46 (2004), pp. 957-979.

[11] V.V. Barve, O.A. Ezekoye and N.T. Clemens, Numerical study of evolution of strongly forced axisymmetric laminar cold-flow jets, AIAA J. 44 (2006), pp. 1976-1752.

[12] N. Riley, M. Sánchez-Sanz and E. Watson, A planar unsteady jet, J. Fluid Mech. 638 (2009), pp. 161-172.

[13] W.G. Bickley, The plane jet, Phil. Mag. 23 (1937), pp. 727-731.

[14] K.C. Smyth, J.E. Harrington, E.L. Johnson and W.M. Pitts, Greatly enhanced soot scattering in fickering $\mathrm{CH}_{4} /$ air diffusion flames, Combust. Flame 95 (1993), pp. 229-239.

[15] C.R. Kaplan, C.R. Shaddix and K.C. Smyth, Computations of enhanced soot production in time-varying $\mathrm{CH}_{4}$ /air diffusion flames, Combust. Flame 106 (1996), pp. 392-405.

[16] R.R. Skaggs and J.H. Miller, Tunable diode laser absorption measurements of carbon monoxide and temperature in a time-varying methane-air non-premixed flame, Proc. Combust. Inst. 26 (1996), pp. 1181-1188.

[17] R.K. Mohammed, M.A. Tanoff, M.D. Smooke, A.M. Schaffer and M.B. Long, Computational and experimental study of a forced, time-varying, axisymmetric, laminar diffusion flame, Proc. Combust. Inst. 27 (1998), pp. 693-702.

[18] R.B. Pember, L.H. Howell, J.B. Bell, P. Colella, W.Y. Crutchfield, W.A. Fiveland and J.P. Jessee, An adaptive projection method for unsteady, low-Mach number combustion, Combust. Sci. Tech. 140 (1998), pp. 123-168.

[19] M.S. Day and J.B. Bell, Numerical simulation of laminar reacting flows with complex chemistry, Combust. Theor. Model. 4 (2000), pp. 535-556.

[20] B.A.V. Bennett and M.D. Smooke, Unsteady axisymmetric laminar diffusion flames: an application of local rectangular refinement, Eastern States Section of the Combustion Institute, Fall Technical Meeting (Hilton Head, South Carolina, 2001), pp. 332-335.

[21] S.B. Dworkin, B.C. Connelly, A.M. Schaffer, B.A.V. Bennett, M.B. Long, M.D. Smooke, M.P. Puccio, B. McAndrews and J.H. Miller, Computational and experimental study of a forced, time-dependent, methane-air coflow diffusion flame, Proc. Combust. Inst. 31 (2007), pp. 971978.

[22] S.B. Dworkin, B.A.V. Bennett and M.D. Smooke, A mass-conserving vorticity-velocity formulation with application to nonreacting and reacting flows, J. Comput. Phys. 215 (2006), pp. $430-447$.

[23] C.R. Wilke, A viscosity equation for gas mixtures, J. Chem. Phys. 18 (1950), pp. 517-519.

[24] J.R. Andrews and O. Biblarz, Temperature dependence of gas properties in polynomial form, Naval Postgraduate School Report NPS67-81-001 (1981).

[25] R.J. Hall, The radiative dissipation in planar gas-soot mixtures, J. Quant. Spectrosc. Radiat. Transfer 51 (1994), pp. 635-644.

[26] C. Pantano, Direct simulation of non-premixed flame extinction in a methane-air jet with reduced chemistry, J. Fluid Mech. 514 (2004), pp. 231-270.

[27] F.A. Williams, Combustion Theory, Addison-Wesley, Reading, 1985.

[28] M.D. Smooke and V. Giovangigli, Reduced Kinetic Mechanisms and Asymptotic Approximations for Methane-Air Flames Springer-Verlag, Berlin, 1991, Chap. 1, pp. 1-28. 
[29] M.D. Smooke, R.E. Mitchell and D.E. Keyes, Numerical solution of two-dimensional axisymmetric laminar diffusion flames, Combust. Sci. and Tech. 67 (1989), pp. 85-122.

[30] K.T. Walsh, J. Fielding, M.D. Smooke, M.B. Long and A. Liñán, A comparison of computational and experimental lift-off heights of coflow laminar diffusion flames, Proc. Combust. Inst. 30 (2005), pp. 357-365.

[31] E. Fernández-Tarrazo and M. Vera, A. Liñán, Liftoff and blowoff of a diffusion flame between parallel streams of fuel and air, Combust. Flame 144 (2006), pp. 261-276.

[32] A. Ern and M.D. Smooke, Vorticity-velocity modeling for three-dimensional compressible flows, J. Comput. Phys. 105 (1993), pp. 58-71.

[33] S.B. Dworkin, J.A. Cooke, B.A.V. Bennett, B.C. Connelly, M.B. Long, M.D. Smooke, R.J. Hall and M.B. Colket, Distributed-memory parallel computation of a forced, time-dependent, sooting, ethylene'air coflow diffusion flame, Combust. Theory Model. 13 (2009), pp. 795-822.

[34] P. Deuflhard, A modified Newton method for the solution of ill-conditioned systems of nonlinear equations with applications to multiple shooting, Numer. Math. 22 (1974), pp. 289-315.

[35] M.D. Smooke, Error estimate for the modified Newton method with applications to the solution of nonlinear; two-point boundary value problems, J. Optimiz. Theory Appls. 39 (1983), pp. $489-511$.

[36] B.A.V. Bennett and M.D. Smooke, Local rectangular refinement with application to axisymmetric laminar flames, Combust. Theory Model. 2 (1998), pp. 221-258.

[37] B.A.V. Bennett, J. Fielding, R.J. Mauro, M.B. Long and M.D. Smooke, A comparison of the structures of lean and rich axisymmetric laminar Bunsen flames: application of local rectangular refinement solution-adaptive gridding, Combust. Theory Model. 3 (1999), pp. 657-687.

[38] B.A.V. Bennett, C. McEnally, L. Pfefferle, M.D. Smooke and M. B. Colket, Computational and experimental study of axisymmetric coflow partially premixed ethylene/air flames, Combust. Flame 127 (2001), pp. 2004-2022.

[39] B.A.V. Bennett, Z. Cheng, R.W. Pitz and M.D. Smooke, Computational and experimental study of oxygen-enhanced axisymmetric laminar methane flames, Combust. Theory Model. 12 (2008), pp. 497-527.

[40] H.A. van der Vorst, Bi-CGSTAB: A fast and smoothly converging variant of Bi-CG for the solution of nonsymmetric linear systems, SIAM J. Sci. Stat. Comput. 13 (1992), pp. 631-644.

[41] A. Ern, V. Giovangigli, D.E. Keyes and M.D. Smooke, Towards polyalgorithmic linear system solvers for nonlinear elliptic problems, SIAM J. Sci. Stat. Comput. 15 (1994), pp. 681-703.

[42] A. Ern, Vorticity-velocity modeling of chemically reacting flows, $\mathrm{PhD}$ thesis, Yale University, New Haven, Connecticut, USA (1994).

[43] B.A.V. Bennett, C.S. McEnally, L.D. Pfefferle, M.D. Smooke and M.B. Colket, Computational and experimental study of the effects of adding dimethyl ether and ethanol to nonpremixed ethylene/air flames, Combust. Flame 156 (2009), pp. 1289-1302.

[44] B.A.V. Bennett, Adaptive numerical modeling of natural convection, conduction, and solidifcation within mold cavities, Numer. Heat Transfer Part A 51 (2007), pp. 313-342.

[45] J. Buckmaster and N. Peters, The infinite candle and its stability - a paradigm for flickering diffusion flames, Proc. Combust. Inst. 21 (1986), pp. 1829-1836.

[46] D.S. Chamberlin and A. Rose, The ficker of luminous flames, Proc. Combust. Inst. 1 (1928), pp. 27-32.

[47] B.W. Albers and A.J. Agrawal, Schlieren analysis of an oscillating gas-jet diffusion flame, Combust. Flame 119 (1999), pp. 84-94.

[48] M.Y. Bahadori, L. Zhou, D.P. Stocker and U. Hegde, Functional dependence of flame flicker on gravitational level, AIAA J. 39 (2001), pp. 1404-1406.

[49] H. Sato, G. Kushida, K. Amagai and M. Arai, Numerical analysis of the gravitational effect on the buoyancy-driven fluctuations in diffusion flames, Proc. Combust. Inst. 20 (2002), pp. 1671-1678.

[50] Y. Xu, M.D. Smooke, P. Lin and M.B. Long, Primitive variable modeling of multidimensional laminar flames, Combust. Sci. Tech. 90 (1993), pp. 298-313.

[51] M.D. Smooke, A. Ern, M.A. Tanoff, B.A. Valdati, R.K. Mohammed, D.F. Marran and M.B. Long, Computational and experimental study of $N O$ in an axisymmetric laminar diffusion flame, Proc. Combust. Inst. 26 (1996), pp. 2161-2170.

[52] J.C. Lasheras, A. Liñán, A. Lecuona and P. Rodríguez, Vorticity dynamics in three-dimensional pulsating coflowing jet diffusion flames, Proc. Combust. Inst. 24 (1992), pp. 321-332. 
[53] T.C. Williams, C.R. Shaddix, R.W. Schefer and P. Desgroux, The response of buoyant laminar diffusion flames to low-frequency forcing, Combust. Flame 151 (2007), pp. 676-684.

[54] J.C. Sheu, D.P. Stocker and L.D. Chen, Dynamic response of a pulsed Burke-Schumann diffusion flame, Third Inter. Microg. Comb. Workshop. NASA CP-10174 (1995), pp. 333-338.

[55] M. Thuillard, A new flame detector using the latest research on flames and fuzzy-wavelet algorithms, Fire Res. J. 37 (2002), pp. 371-380.

[56] H.G. Im and J.H. Chen, Effects of flow strain on triple flame propagation, Combust. Flame 126 (2001), pp. 1384-1392.

[57] K.C. Smyth, P.J.H. Tjossem, A. Hamins and J.H. Miller, Concentration measurements of OH and equilibrium analysis in a laminar methane-air diffusion flame, Combust. Flame 79 (1990), pp. 366-380.

[58] D.A. Everest, C.R. Shaddix and K.C. Smyth, Quantitative two-photon laser-induced fluorescence imaging of $\mathrm{CO}$ in flickering $\mathrm{CH}_{4}$-air diffiusion flames, Proc. Combust. Inst. 26 (1999), pp. $1161-1169$

[59] N. Peters, Numerical Simulation of Combustion Phenomena, Springer, Berlin, 1985.

[60] N. Peters and R.J. Kee, The computation of stretched laminar methane-air diffusion flames using a reduced four-step mechanism, Combust. Flame 68 (1987), pp. 17-29. 